\title{
ON SETS THAT ARE UNIQUELY DETERMINED BY A RESTRICTED SET OF INTEGRALS
}

\author{
J. H. B. KEMPERMAN
}

\begin{abstract}
In many applied areas, such as tomography and crystallography, one is confronted by an unknown subset $S$ of a measure space $(X, \lambda)$ such as $\mathbf{R}^{n}$, or an unknown function $0 \leq \phi \leq 1$ on $X$, having known moments (integrals) relative to a specified class $F$ of functions $f: X \rightarrow \mathbf{R}$. Usually, these $F$-moments do not fully determine the object $S$ or function $\phi$. We will say that $S$ is a set of uniqueness if no other function $0 \leq \psi \leq 1$ has the same $F$-moments as $S$ in so far as the latter moments exist. Here, $S$ is identified with its indicator function.

Within this general setting and with no further assumptions, we develop a powerful sufficient condition for uniqueness, called generalized additivity. When $F$ is a finite class, this condition of generalized additivity is shown to be also necessary for uniqueness. For each $\phi$, which is not the indicator function of a set of uniqueness, there exist infinitely many sets having the same $F$ moments as $\phi$, provided $(X, \lambda, F)$ is nonatomic or regular and, moreover, 'strongly rich', a condition which is satisfied in many applications.

Using such general results, we also study the uniqueness problem for measures with given marginals relative to a given system of projections $\pi_{j}: X \rightarrow Y_{j}$ $(j \in J)$. Here, one likes to know, for instance, what subsets $S$ of $X$ are uniquely determined by the corresponding set of projections ( $X$-ray pictures). It is allowed that $\lambda(S)=\infty$. Our results are also relevant to a wide class of optimization problems.
\end{abstract}

\section{INTRODUCTION}

Let $X$ be a measurable space and $\lambda$ a fixed nonzero $\sigma$-finite reference measure on $X$. All sets and functions below are assumed to be measurable. Sets or functions which are equal a.e. [ $\lambda]$ will be identified. A subset $S$ of $X$ can be identified with the associated measure $\mu_{S}(B)=\lambda(B \cap S)$ on $X$. Thus, $d \mu_{S}=1_{S} d \lambda$, where the so-called indicator function $1_{S}(x)$ has the value 1 or 0 , respectively, depending on whether $x \in S$ or $x \notin S$, respectively.

Received by the editors November 28, 1988.

1980 Mathematics Subject Classification (1985 Revision). Primary 44A60, 68P20, 28A35, 49D35, 49A55, 49B36.

Key words and phrases. Sets with given moments, generalized additive sets, sets of uniqueness, reconstructing a set from its projections, strongly rich systems, optimal bang-bang control, tomography.

This research was supporied in part by the National Science Foundation Grant DMS- 8802557 and by Bellcore, Inc. 
1.1. For any function $f: X \rightarrow \mathbf{R}$, the quantity $d_{f}$ defined by

$$
d_{f}=d_{f}(S)=\int_{X} f d \mu_{S}=\int_{S} f d \lambda
$$

is called a moment of $\mu_{S}$ or $S$, provided $\int_{S}|f| d \lambda<\infty$. If $f=1_{B}$ then $d_{f}=\mu_{S}(B)=\lambda(B \cap S)$. If $X=\mathbf{R}^{3}$ with ordinary Lebesgue measure $\lambda$ then $S$ may be regarded as an object of density 1 . Possible moments $d_{f}(S)$ for this case would be the different moments of inertia of the object $S$ or else the mass $\lambda(B \cap S)$ of $S$ within certain sets $B$.

Let $F$ be a given set of functions $f: X \rightarrow \mathbf{R}$. The triplet $(X, \lambda, F)$ will also be referred to as a system. For $S$ as a subset of $X$, let $F(S)$ be the set of all $f \in F$ such that $\int_{S}|f| d \lambda<\infty$. We like to know what subsets $S$ of $X$ are uniquely determined by its $F$-moments $d_{f}(S)$ is so far as these exist, that is, in so far as $f \in F(S)$. This problem has been studied in detail for special cases, see $\S 2.8$ for further references. The following definitions of uniqueness go back to Fishburn et al. [3, 4]; Gutmann et al. [5] and Kemperman [11], who largely restricted themselves to projection type problems, see $\S 6$ for a general treatment.

1.3. Definition. We call $S$ a weak set of uniqueness, relative to the system $(X, \lambda, F)$, if no other set $T$ exists such that $F(T) \supset F(S)$ and $d_{f}(S)=d_{f}(T)$, for all $f \in F(S)$. In many applications, one is only interested in sets $S$ such that $F(S)=F$, in which case $F(T) \supset F(S)$ would mean that also $F(T)=F$.

Let $M_{0}$ be the set of all functions $\psi: X \rightarrow \mathbf{R}$ satisfying $0 \leq \psi(x) \leq 1$, for all $x \in X$. We will be interested in measures $\mu \leq \lambda$ on $X$, that is, measures of the form $d \mu=\phi d \lambda$ with $\phi \in M_{0}$. Let $F(\phi)$ denote the set of all $f \in F$ such that $\int|f| \phi d \lambda<\infty$. Thus, $F(S)=F\left(1_{S}\right)$ and $F(0)=F$. In many applications each $f \in F$ is $\lambda$-integrable, that is $F(1)=F$, and then $F(\phi)=F$ for all $\phi \in M_{0}$, while $F(S)=F$ for all subsets $S$ of $X$.

A function $\phi \in M_{0}$ is said to be a uniqueness function (relative to the given system $(X, \lambda, F))$ if there exist no other $\psi \in M_{0}$ such that $F(\psi) \supset F(\phi)$ and $d_{f}(\phi)=d_{f}(\psi)$, for all $f \in F(\phi)$. Here, $d_{f}(\phi)$ is the moment defined by

$$
d_{f}(\phi)=\int_{X} f \phi d \lambda \text { when } f \in F(\phi) .
$$

Roughly speaking, $0 \leq \phi \leq 1$ is a uniqueness function if there exist no other $0 \leq \psi \leq 1$ with the same $F$-moments.

A subset $S$ of $X$ is said to be a set of uniqueness if $\phi=1_{S}$ is a uniqueness function. A weak set of uniqueness $S$ can fail to be a set of uniqueness due to the existence of a nonindicator function $\phi \in M_{0}$ such that $\phi d \lambda$ has the same moments as $1_{S} d \lambda$, relative to all $f \in F(S)$. See 2.11 for a simple example.

When $F$ is empty or $F=\{0\}$, we say that $F$ and $(X, \lambda, F)$ are trivial. The system $(X, \lambda, F)$ is said to be singular (and regular, otherwise), if there exists a subset $D$ of $X$ with $\lambda(D)>0$ such that $f(x)=0$ a.e. [ $\lambda]$ on $D$, for 
each $f \in F$. When $F$ is trivial this happens with $D=X$. For each such set $D$, the moments $d_{f}(\phi) \quad(f \in F)$ remain unchanged when one arbitrarily modifies the values $\phi(x) \quad(x \in D)$, subject to $0 \leq \phi \leq 1$. Consequently, uniqueness functions or (weak) sets of uniqueness can only exist when $(X, \lambda, F)$ is regular, in particular, $F$ must be nontrivial.

1.5. General additivity. Below, we introduce the notion of an $s$-additive set or function, relative to the given system $(X, \lambda, F)$. See [11] for a related notion of additivity of degree $s$. A generalized additive set or function is one which is $s$-additive for some choice of $s$. From Theorem 3.17, generalized additivity is a sufficient condition for uniqueness. If $F$ is finite or else $\operatorname{dim} F<\infty$ then generalized additivity is also a necessary condition for uniqueness, see Theorem 4.16. If $H$ is any set of functions $f: X \rightarrow \mathbf{R}$ then $H^{*}$ denotes its linear span and $\operatorname{dim} H=\operatorname{dim} H^{*} \leq \infty$ the corresponding linear dimension.

1.6. Let $s \geq 0$ be an integer. A subset $S$ of $X$ is said to be a special s-additive set (or also a special generalized additive set) if $S$ is of the form

$$
S=\bigcup_{r=0}^{s}\left\{x \in X: f_{q}(x)=0 \text { for } 0 \leq q<r ; f_{r}(x)>0\right\},
$$

for some choice of the functions $f_{r} \in F(S)^{*} \quad(r=0,1, \ldots, s)$, such that $\lambda(Z)=0$, where

$$
Z=\left\{x \in X: f_{r}(x)=0, \text { for all } r=0, \ldots, s\right\} .
$$

The integer $s$ and functions $f_{r} \in F(S)^{*}$ are not unique.

A function $\phi \in M_{0}$ is said to be an s-additive function (or also a generalized additive function) if functions $f_{r} \in F(\phi)^{*} \quad(r=0, \ldots, s)$ can be found such that $\phi(x)=1$ if $x \in S$; further $0 \leq \phi(x) \leq 1$ for $x \in Z$ and $\phi(x)=0$ for $x \notin S \cup Z$, while moreover $F(\phi)$ is $Z$-determining, (as is trivially true when $\lambda(Z)=0)$. Here, $S$ and $Z$ are again as in (1.7), (1.8). Choosing $f_{s+1}=f_{s}$, this implies that $\phi$ is also $(s+1)$-additive.

Here, a set $H$ of functions $f: X \rightarrow \mathbf{R}$ is said to be $Z$-determining if there does not exist any nonzero bounded function $\theta$ supported by $Z$ (that is, $\theta(x)=$ 0 when $x \notin Z$ ), which is orthogonal to $H$, in the sense that $\int|f \theta| d \lambda<\infty$ and $\int f \theta d \lambda=0$ for all $f \in H$. Naturally, this is the same as $H^{*}$ being $Z$-determining.

We will say that $\phi$ is a special generalized additive function when by a suitable choice of $s$ and the $f_{r} \in F(\phi)^{*} \quad(r=0, \ldots, s)$ the above is true with $\lambda(Z)=0$. This is the same as saying that $\phi$ is the indicator function of a special generalized additive set, as defined above.

It is not excluded that $Z=X$, equivalently, that $f_{r}=0(r=0, \ldots, s)$. Naturally, in that case, $F(\phi)$ is required to be $X$-determining. Functions $\phi \in M_{0}$ of this type will also be said to be $s$-additive with $s=-1$. They only exist when $F$ itself is $X$-determining. If all $f \in F$ are $\lambda$-integrable this 
would mean that each $\phi \in M_{0}$ is a uniqueness function, a rather uninteresting situation as far as the present uniqueness problem is concerned.

A subset $T$ of $X$ is said to be $s$-additive if and only if its indicator function $\phi=1_{T}$ is an $s$-additive function. This means that $f_{r} \in F(T)^{*} \quad(r=0, \ldots, s)$ can be found such $S \cup Z \supset T \supset S$ while $F(T)$ is $Z$-determining. Here, $S$ and $Z$ are as above. If $F(T)$ is $X$-determining, one could choose $f_{r}=0$ for all $r$, in which case $S$ is empty and $Z=X$.

1.9. Additive sets. A set $S$ is said to be additive if $f_{0}, f^{+} \in F(S)^{*}$ can be found such that either $S=\left\{x \in X: f_{0}(x)>0\right\}$ or $S=\left\{x \in X: f_{0}(x) \geq 0\right\}$ and, moreover, $f^{+}(x)>0$ a.e. [ $[\lambda]$ on the zero-set $\left\{x \in X: f_{0}(x)=0\right\}$.

Choosing $f_{1}= \pm f^{+}$, we see that each additive set is also a special 1-additive set; (the converse is false, see 3.13). In many applications, the above $f^{+}$. condition is trivially satisfied, because of the existence of a $\lambda$-integrable function $f^{+} \in F$, (hence, $f^{+} \in F(S)$, for all $S$ ), such that $f^{+}(x)>0$ a.e. [ $\lambda$ ] on $X$. If $1 \in F$ and $\lambda(X)<\infty$ then $f^{+}=1$ will do.

In special cases, an analogous but somewhat more restricted notion of additive set was employed by Krein and Nudel'man [14, 15]; Fishburn, Lagarias, Reeds, and Shepp [3, 4]; see also [5, 11].

1.10. Remarks. As to (1.7), (1.8), if in the sequence $\left\{f_{0}, \ldots, f_{s}\right\}$ one drops those $f_{r}$ which depend linearly on the $f_{q}$ with $0 \leq q<r$, and thereby lowers the value of $s$, then the associated sets $S$ and $Z$ remain unchanged. Hence, assuming that $s$ is minimal (as we may), it follows that the resulting set of functions $f_{r}(r=0, \ldots, s)$ is ( $\lambda$-essentially) linearly independent. Hence, $s<\operatorname{dim} F$, while each $f_{r}$ is not zero a.e. [ $\lambda$ ]. The case $s=-1$ (no remaining function at all and $Z=X$ ) corresponds to the situation where $F(\phi)$ or $F(S)$ is $X$-determining.

As was already mentioned, a sufficient condition for a set $S$ to be a set of uniqueness, or a function $\phi \in M_{0}$ to be a uniqueness function, respectively, is that $S$ be a generalized additive set, or $\phi$ a generalized additive function, respectively. On the other hand, we observed in 1.4 that there cannot exist any uniqueness function if $(X, \lambda, F)$ is singular or even trivial. Hence, in this case there cannot exist any generalized additive function either. And indeed in that case it is impossible that $F(\phi)$ is $Z$-determining. After all, $Z$ as defined by (1.8) contains the set $D$ in 1.4. Thus $\lambda(D)>0$, while each $f \in F$ vanishes a.e. on $D$. Any bounded function $\theta$ supported by $D$ (and thus by $Z$ ) is orthogonal to $F$ and thus orthogonal to $F(\phi)$, showing that $F(\phi)$ is not $Z$-determining.

1.11. The case $\operatorname{dim} F<\infty$. Let us now consider in more detail the situation that $m=\operatorname{dim} F<\infty$, which occurs in many applications. Thus $F^{*}$ is spanned by a finite set $\left\{f_{1}, \ldots, f_{m}\right\}$ of ( $\lambda$-essentially) linearly independent functions. For this important case, and with no further assumptions, Theorem 4.16 states that in order that $\phi \in M_{0}$ be a uniqueness function, it is not only sufficient but also necessary that $\phi$ be a generalized additive function. That is, $\phi$ must be 
$s$-additive for some $s$. In view of Remark 1.10, one can always achieve that $s \leq m-1$.

1.12. Part of the definition of $s$-additivity requires that $F(\phi)$ be $Z$-determining with $Z$ as in (1.8), as is trivially true when $\lambda(Z)=0$. Suppose $\lambda(Z)>0$ and further (for the moment) that each $f \in F$ is $\lambda$-integrable. Then $\phi$ remains $s$-additive (and thus a uniqueness function) when one arbitrarily modifies the values $\phi(x) \quad(x \in Z)$, subject to $0 \leq \phi \leq 1$. Hence, in this situation there exist many uniqueness functions $\psi \in M_{0}$ which are not indicator functions.

If $m=\operatorname{dim} F<\infty$ and $F(\phi)$ is $Z$-determining with $\lambda(Z)>0$, then the restriction $\lambda$ to $Z$ must be purely atomic with a finite number $k$ of atoms, $1 \leq k \leq m$. Moreover, the restriction of $F(\phi)$ to $Z$ has its dimension equal to $k$.

1.13. Next, consider the case that $(X, \lambda)$ is nonatomic and further $\operatorname{dim} F<\infty$. In view of the latter remark, $F(\phi)$ can be $Z$-determining only when $\lambda(Z)=$ 0 , (thus, the case $s=-1$ in 1.6 never occurs). Consequently, $\phi \in M_{0}$ is a uniqueness function if and only if it is of the form $\phi=1_{S}$ with $S$ as a special generalized additive subset of $X$; (that a uniqueness function must be an indicator function is also easily seen directly, see Lemma 4.4).

As a corollary, we have that the empty set is a set of uniqueness if and only if there exist one or more linearly independent (nonzero) functions $f_{0}, \ldots, f_{s} \in$ $F^{*}$, such that $Z$ defined by (1.8) is a $\lambda$-null set, and that further the first nonzero element in the sequence $\left\{f_{0}(x), \ldots, f_{s}(x)\right\}$ is strictly negative, for almost [ $\lambda]$ all $x \in X$. Similarly, for $S=X$ to be a set of uniqueness, except that now we only allow $f_{r} \in F(X)^{*}$, that is, each $f_{r}$ must be a finite linear combination of $\lambda$-integrable functions $f_{r, j} \in F$.

1.14. Finally, consider the case that $(X, \lambda)$ is nonatomic and $\operatorname{dim} F<\infty$ while, moreover, each nonzero $f \in F$ satisfies $\lambda\left(Z_{f}\right)=0$, where $Z_{f}=\{x \in$ $X: f(x)=0\}$. Under these conditions, Theorem 4.11 asserts that a function $0 \leq \phi \leq 1$ is a uniqueness function if and only if $\phi$ is an indicator function $\phi=1_{S}$ with $S$ of the form $S=\left\{x \in X: f_{0}(x)>0\right\}$ and $f_{0}$ as a nonzero member of $F(S)^{*}$, hence $\int\left(f_{0}\right)_{+} d \lambda<\infty$.

For instance, under the assumptions stated, the empty set is a set of uniqueness if and only if $f^{+}>0$ (a.e. [ $\left.\lambda\right]$ ) for some $f^{+} \in F^{*}$. Similarly, $S=X$ is a set of uniqueness if and only if the latter $f^{+}$can be chosen as a finite linear combination of $\lambda$-integrable functions $f_{j} \in F$.

Theorem 4.11 admits many applications. The property $\lambda\left(Z_{f}\right)=0$ is often satisfied, for instance, when $X$ is an open subset of $\mathbf{R}^{n}$ supplied with Lebesgue measure $\lambda$ and, moreover, each $f \in F$ is analytic on $S$. Another important special case, where $X$ is a real interval and $F$ is spanned by a Tchebycheff system, goes back to Ahiezer and Krein [1]; Karlin and Studden [7]; Krein and Nudel'man $[14,15]$. 
1.15. Richness. In $\S 5$, given $\phi \in M_{0}$, we investigate the possibly empty collection of sets $S$ such that $d \mu_{S}={ }_{S} d \lambda$ has the same $F$-moments as $d \mu=\phi d \lambda$. For each $\phi \in M_{0}$, let $M_{0}(\phi)$ consis: of all $\psi \in M_{0}$ satisfying $F(\psi) \supset F(\phi)$ and

$$
\int f \psi d \lambda=\int f \phi d \lambda, \quad \text { for all } f \in F(\phi)=\left\{f \in F: \int|f| \phi d \lambda<\infty\right\} .
$$

In particular, $\phi \in M_{0}(\phi)$. Thus, $\phi$ is a uniqueness function if and only if $M_{0}(\phi)=\{\phi\}$. For $S$ as a subset of $X$, we put $M_{0}(S)=M_{0}\left(1_{S}\right)$. Thus, $S$ is a weak set of uniqueness if $M_{0}(S)$ contains no indicator function $1_{T}$ other than $1_{S}$. And $S$ is a set of uniqueness if $M_{0}(S)=\left\{1_{S}\right\}$.

We will say that the system $(X, \lambda, F)$ is rich if each set $M_{0}(\phi)$ contains at least one indicator function. And $k$-rich if for each nonindicator function $\phi \in M_{0}$ there are at least $k$ different sets $T$ such that $1_{T} \in M_{0}(\phi)$. Here, $1 \leq k \leq+\infty$ is fixed. Equivalently, $(X, \lambda, F)$ is $k$-rich if $M_{0}(\phi)$ contains at least $k$ distinct indicator functions, for each $\phi \in M_{0}$ not of the form $\phi=1_{S}$ with $S$ as a set of uniqueness. Hence, $k$-rich implies 1 -rich which is the same as rich. If $(X, \lambda, F)$ is 2-rich then each weak set of uniqueness is also a set of uniqueness.

If $(X, \lambda)$ is a finite discrete space and $F$ is nontrivial then $(X, \lambda, F)$ cannot possibly be rich, simply because $X$ has only finitely many different subsets. On the other hand, $(X, \lambda, F)$ is always rich when $\operatorname{dim} F<\infty$ and $(X, \lambda)$ is nonatomic, as follows easily from Lyapunov's theorem, see 5.2.

1.16. Definition. The system $(X, \lambda, F)$ is said to be strongly rich if, for each choice of $\phi \in M_{0}$ and the subset $D$ of $X$ with $\lambda(D)>0$, there exists a nonzero bounded function $\theta$ on $X$ which is supported by $D$ and, moreover, is orthogonal to $F(\phi)$, see (5.6). Equivalently, $\lambda(D)>0$ implies that $F(\phi)$ is not $D$-determining, (see 1.6). Obviously, this property is maintained when $F$ is replaced by a smaller set.

If $(X, \lambda, F)$ is strongly rich and, moreover, regular then it must be nonatomic. If $(X, \lambda, F)$ is strongly rich and nonatomic then also $(X, \lambda, G)$ is strongly rich, whenever $G$ is contained in the linear span of $F$ and finitely many other functions, see 5.8 . Since $(X, \lambda,\{0\})$ is trivially strongly rich, this implies that $(X, \lambda, F)$ is strongly rich as soon as $(X, \lambda)$ is nonatomic and $\operatorname{dim} F<\infty$.

In a different context, the notion 'strongly rich' (under a different name) already occurs in Kingman and Robertson [13]. The above terms 'rich' and 'strongly rich' were first used in Gutmann et al. [5].

1.17. Suppose $(X, \lambda, F)$ is strongly rich. Then the set of extreme points of $M_{0}(\phi)$ coincides with the set of indicator functions $1_{T} \in M_{0}(\phi)$, see Proposition 5.9. Therefore, in order that $(X, \lambda, F)$ be $k$-rich, it is necessary and sufficient that $M_{0}(\phi)$ have at least $k$ extreme points, for each choice of the nonindicator function $\phi \in M_{0}$. The major result here is the useful Theorem 5.15. It asserts that $(X, \lambda, F)$ is $\infty$-rich when $(X, \lambda, F)$ is strongly rich and 
nonatomic and, moreover, each $f \in F$ is $\lambda$-integrable. It should be stressed that this result is very much built on ideas already present in Kingman and Robertson [13] and Gutmann et al. [5]. It has important consequences, see Theorems 5.20 and 6.23 .

1.18. Reconstructing a set from its projections. Let $(X, \lambda)$ be a $\sigma$-finite measure space and let $\pi=\left\{\pi_{j} ; j \in J\right\}$ be a given nonempty system of mappings $\pi_{j}: \rightarrow$ $Y_{j}$ where $Y_{j}$ is a measurable space. $\S 6$ is devoted to applications of the previous results to measures $\mu$ on $X$ with given $\pi$-marginals $\pi_{j} \mu \quad(j \in J)$. For each $\phi \in M_{0}$, let $M_{00}(\phi)$ be the set of all $\psi \in M_{0}$ such that $d \mu_{\psi}=\psi d \lambda$ has precisely the same $\pi$-marginals as $d \mu_{\phi}=\phi d \lambda$. If $M_{00}(\phi)=\{\phi\}$ then $\phi$ will be said to be a $\pi$-uniqueness function. That is, $\phi \in M_{0}$ is uniquely determined by $\left\{\pi_{j} \mu_{\phi} ; j \in J\right\}$. A subset $S$ of $X$ is said to be a set of $\pi$-uniqueness if $\phi=1_{S}$ is a $\pi$-uniqueness function.

1.19. In general, it is a very difficult problem to characterize the class of all $\pi$-uniqueness functions. In 6.10 , we discuss the class of so-called special generalized additive sets $S$. Each member of this class is a set of $\pi$-uniqueness and most known examples of a set of $\pi$-uniqueness do belong to this class. Here, a special generalized additive set is defined in the usual way (see 1.6) in terms of finitely many functions $f_{r}: X \rightarrow \mathbf{R}(r=0, \ldots, s)$, each of which is a finite sum of functions $g_{j}\left(\pi_{j} x\right)$ on $X$, where $g_{j}: Y_{j} \rightarrow \mathbf{R}$ must be such that

$$
\int_{S}\left|g_{j}\left(\pi_{j} x\right)\right| \lambda(d x)<\infty .
$$

1.20. For any fixed $1 \leq k \leq \infty$, we say that $(X, \lambda)$ is $k$-rich relative to $\pi$ when $M_{00}(\phi)$ contains at least $k$ distinct indicator functions, for all $\phi \in M_{0}$ not of the form $\phi=1_{S}$ with $S$ as a set of $\pi$-uniqueness. When $k=1$ we also speak of $(X, \lambda)$ as being rich relative to $\pi$. It implies that all $\pi$-uniqueness functions (if any) must be indicator functions.

We say that $(X, \lambda)$ is strongly rich relative to $\pi$ if, for each subset $D$ of $X$ with $0<\lambda(D)<\infty$, there exists a nonzero bounded function $\theta$ supported by $D$, such that the signed measure $d \eta=\theta d \lambda$ satisfies $\pi_{j} \eta=0$, for all $j \in J$.

Each of the above richness properties is preserved when replacing the system $\pi=\left\{\pi_{j} ; j \in J\right\}$ by a derived (or induced) system $\tau=\left\{\tau_{j} ; j \in J\right\}$ where $\tau_{j}: X \rightarrow Z_{j}$ is of the form $\tau_{j}=\sigma_{j} \pi_{j}$ with $\sigma_{j}: Y_{j} \rightarrow Z_{j}$, for all $j \in J$; see 6.25 .

1.21. The central Theorem 6.23 states that if $(X, \lambda)$ is strongly rich relative to $\pi$ then $(X, \lambda)$ is nonatomic and $\infty$-rich relative to $\pi$; in particular, any $\pi$-uniqueness function is an indicator function. And similarly when $\pi$ is replaced by any derived system $\tau$.

1.22. As was shown in $[5,11]$, the measure space $(X, \lambda)$ is strongly rich relative to $\pi$ in the important situation, also occurring in tomography, where $X=$ $\mathbf{R}^{n}$ with $\lambda$ as any absolutely continuous measure (relative to $n$-dimensional 
Lebesgue measure $\left.m_{n}\right)$, while $\pi=\left(\pi_{j} ; j \in J\right)$ consists of finitely many central projections. We do allow the center $P_{j}$ of $\pi_{j}$ to be a point at infinity, in which case $\pi_{j}$ is to be interpreted as a parallel projection along lines of direction $P_{j}$. This setup admits many different derived systems $\tau=\left\{\tau_{j}=\sigma_{j} \pi_{j} ; j \in J\right\}$, for instance, $\tau=\left\{\tau_{1}, \ldots, \tau_{n}\right\}$ with $\tau_{j} x=x_{j}$ as the $j$ th coordinate of $x \in \mathbf{R}^{n}$ $(j=1, \ldots, n)$. See 6.35 for related results in the literature.

1.23. Optimization. The results of the present paper are also relevant to the frequently occurring optimization problem, where one tries to maximize $Q(\phi)=$ $\int h \phi d \lambda$, with $\phi: X \rightarrow \mathbf{R}$ as an arbitrary function satisfying $0 \leq \phi \leq 1$ and, moreover, any finite or infinite number of prescribed moment conditions $\int f \phi d \lambda=d_{f}(f \in F)$. An additional condition, that $d \mu=\phi d \lambda$ have given marginals $\pi_{j} \mu=\nu_{j}(j \in J)$, can also be expressed as a set of such moment conditions, see 6.36. The notion of a generalized additive set or function leads to sufficient conditions in order that an optimal function $\phi$ be the only one. These conditions are also necessary when $F$ or $\operatorname{dim} F$ is finite. If $\phi: X \rightarrow\{0,1\}$ is an indicator function then the function $\phi$ is often described as a 'bang-bang' control. In particular, the above richness conditions lead to sufficient conditions in order that there exists at least one or even infinitely many optimal bang-bang controls. See 2.22, 4.27, 5.20, and 6.25 for further details.

\section{Definitions and basic Results}

In the sequel, $X$ is a fixed measurable space supplied with a nonzero $\sigma$-finite reference measure $\lambda$. All subsets of $X$ and functions on $X$, considered below, are assumed to be measurable. Functions on $X$ (or subsets of $X$ ) which are equal a.e. [ $\lambda]$ will be identified. The indicator function of a subset $S$ of $X$ is denoted as $1_{S}$. Thus, $1_{S}(x)=1$ or 0 , respectively, depending on whether $x \in S$ or $x \in S^{c}=X / S$, respectively. Sometimes, as will be clear from the context, the symbol 1 denotes the function $f_{0}(x) \equiv 1$ on $X$. Unspecified integrals extend over all of $X$. Let further

$$
M_{0}=\{\text { all } \phi: X \rightarrow \mathbf{R} \text { such that } 0 \leq \phi(x) \leq 1(x \in X)\},
$$

thus $1_{S} \in M_{0}$ for all $S$. The class of all measures $d \mu=\phi d \lambda$ with $\phi \in M_{0}$ is denoted as $M$. Equivalently, $M$ is the class of all measures $\mu$ on $X$ such that $\mu \leq \lambda$.

We are interested in functions $\psi: X \rightarrow \mathbf{R}$ that are uniquely determined by bounds $a(x) \leq \psi(x) \leq b(x)$ together with certain moments $\int g \psi d \lambda(g \in G)$. Introducing

$$
h(x)=b(x)-a(x) ; \quad \phi(x)=(\psi(x)-a(x)) / h(x) ; \quad F=\{g h: g \in G\},
$$

the problem largely reduces to a study of functions $\phi \in M_{0}$ which are uniquely determined by the associated collection of moments $\int f \phi d \lambda(f \in F)$. 
2.2. With $H$ as any set of functions $f: X \rightarrow \mathbf{R}$, let $M_{0}[H]$ denote the convex set

$$
M_{0}[H]=\left\{\phi \in M_{0}: \int|f| \phi d \lambda<\infty \text { for all } f \in H\right\} .
$$

To each $\phi \in M_{0}[H]$, we associate the $H$-moment point $c_{H}(\phi) \in \mathbf{R}^{H}$ defined by

$$
c_{H}(\phi)=\left\{d_{f}(\phi): f \in H\right\}, \quad \text { where } d_{f}(\phi)=\int_{X} f \phi d \lambda .
$$

The set of all such points

$$
C_{H}=\left\{c_{H}(\phi): \phi \in M_{0}[H]\right\}
$$

will be referred to as the $H$-moment space. Hence, $C_{H}$ is a convex subset of $\mathbf{R}^{H}$ such that $0=c_{H}(0) \in C_{H}$. For $S$ as a subset of $X$, we write $d \mu_{S}=1_{S} d \lambda$ and $c_{H}(S)=c_{H}\left(1_{S}\right)$.

2.6. All the following considerations are relative to the system $(X, \lambda, F)$ with $F$ as a fixed collection of functions $f: X \rightarrow \mathbf{R}$. If desired, one may assume that $0 \in F$. Given $\phi \in M_{0}$, let $F(\phi)$ be the subset of $F$ defined by

$$
F(\phi)=\left\{f \in F: \int|f| \phi d \lambda<\infty\right\} .
$$

For instance, $F(0)=F$. If $F(\phi)$ is empty or $F(\phi)=\{0\}$ then $F(\phi)$ is said to be trivial. A desirable property is that $F(\phi)=F$ holds for all $\phi \in M_{0}$. This happens if and only if each $f \in F$ is $\lambda$-integrable.

For any subset $H$ of a linear space, let $H^{*}=\operatorname{span} H$ denote the linear span of $H$ (over the reals). It should be noted that $F^{*}(\phi)$ might be strictly larger than $F(\phi)^{*}$. For instance, it might happen, that the finitely many nonzero $\alpha_{j} \in \mathbf{R}$ and $f_{j} \in F$ are such that $\int\left|\sum_{j} \alpha_{j} f_{j}\right| \phi d \lambda<\infty$, though $\int\left|f_{j}\right| \phi d \lambda=\infty$ for all $j$. This is the main reason we did not assume that $F$ is a linear set, (which would simplify some arguments and would imply that $0 \in F$ ). If desired, one may assume without any real loss of generality that $F$ is invariant under scalar multiplication.

Clearly, (2.3), (2.5) are equivalent to

$$
M_{0}[H]=\left\{\phi \in M_{0}: F(\phi) \supset H\right\} \quad \text { and } C_{H}=\left\{c_{H}(\phi): \phi \in M_{0} ; F(\phi) \supset H\right\} \text {. }
$$

For each $\phi \in M_{0}$, let $c(\phi)=c_{G}(\phi) \in \mathbf{R}^{G}$, where $G=F(\phi)$. This 'moment' point $c(\phi)$ represents the values of all the existing moments which $d \mu=\phi d \lambda$ happens to have relative to the different functions $f \in F$.

2.8. Let $\phi \in M_{0}$ and $d \mu=\phi d \lambda$. An important role is played by the associated convex set

$$
M_{0}(\phi)=\left\{\psi \in M_{0}: F(\psi) \supset F(\phi) ; \int f \psi d \lambda=\int f \phi d \lambda, \text { if } f \in F(\phi)\right\} .
$$


Further $M(\mu)$ will denote the set of all corresponding measures $d \nu=\psi d \lambda$; $\psi \in M_{0}(\phi)$. Letting $G=F(\phi)$, the set $M_{0}(\phi)$ thus consists of all $\psi \in M_{0}$ such that, the $G$-moment point $c_{G}(\psi) \in \mathbf{R}^{G}$ is well defined, (that is, $F(\psi) \supset F(\phi)$ ), and moreover coincides with the $G$-moment point $c(\phi)=c_{G}(\phi)$.

Equivalently, $M_{0}(\phi)$ consists of all $0 \leq \psi \leq 1$, such that $\int|f| \psi d \lambda<\infty$ and $\int f \psi d \lambda=\int f \phi d \lambda$, for each $f \in F$ such that $\int|f| \phi \lambda<\infty$, (identifying functions $\psi$ equal a.e. [ $\lambda])$. The smaller the set $F(\phi)$ the larger $M_{0}(\phi)$. For instance $M_{0}(\phi)=M_{0}$ if and only if $F(\phi)$ is trivial. Obviously, $\mu \in M(\mu)$ and $\phi \in M_{0}(\phi)$. Often one assumes that $1 \in F(\phi)$ and then all measures $\nu \in M(\mu)$ have the same finite total mass $\nu(X)=\mu(X)$. It is important to note that

$$
\psi \in M_{0}(\phi) \text { implies } M_{0}(\phi) \supset M_{0}(\psi) \text {. }
$$

After all, suppose $\chi \in M_{0}(\psi)$. Then $F(\chi) \supset F(\psi) \supset F(\phi)$ and moreover, if $f \in F(\phi)$ then $\int f \chi d \lambda=\int f \psi d \lambda=\int f \phi d \lambda$, hence, $\chi \in M_{0}(\phi)$. If $\phi \in M_{0}$ is the indicator function $\phi=1_{S}$ of a subset $S$ of $X$, we also write $c(S)=c\left(1_{S}\right)$ and $F(S)=F\left(1_{S}\right)$, further $M_{0}(S)=M_{0}\left(1_{S}\right)$ and $M(S)=M\left(\mu_{S}\right)$.

For certain special cases, the following notions of uniqueness (see Definitions 2.10 and 2.11) were already studied by Carrington [2]; Fishburn et al. [3, 4]; Gutmann et al. [5]; Karlin and Studden [7, Chapter 8]; Kemperman [11, 12]; Ahiezer, Krein, and Nudel' man [1, p. 70; 14, 15, Chapter 7]; Kuba and Volcic $[16,18]$ and Lorentz [17]. In the older literature $[1,7,14,15]$, the space $X$ is usually a real interval with Lebesgue measure while $F$ is the linear span of a fixed Tchebycheff system on $X$.

2.10. Definition. Let $\phi \in M_{0}$ and $d \mu=\phi d \lambda$. We will say that $\phi$ is a uniqueness function (relative to the system $(X, \lambda, F)$ ) when $M(\mu)=\{\mu\}$, equivalently, when $M_{0}(\phi)=\{\phi\}$.

Equivalently, $0 \leq \phi \leq 1$ is a uniqueness function if for any other $0 \leq \psi \leq 1$, there exists $f \in F$ such that $\int|f| \phi d \lambda<\infty$ and either $\int|f| \psi d \lambda=\infty$ or else $\int f \psi d \lambda \neq \int f \phi d \lambda$.

Roughly speaking, $\phi$ is a uniqueness function if $\phi$ is completely determined by $0 \leq \phi \leq 1$ together with its associated moment point $c(\phi)$. The point $c(\phi)$ represents all the moments $d_{f}(\phi)=\int f \phi d \lambda(f \in F)$ in so far as these moments exist, (see 2.6).

The condition $M_{0}(\phi)=\{\phi\}$ would be impossible if $M_{0}(\phi)=M_{0}$, as happens precisely when $F(\phi)$ is trivial. In particular, when $F$ itself is trivial then the system $(X, \lambda, F)$ does not admit any uniqueness function at all.

Here is an example of a uniqueness function $\phi$ which is not an indicator function. Suppose $(X, \lambda)$ has an atom $T=\left\{x_{0}\right\}$, thus, $\lambda(T)>0$, and let $F$ be the linear span of $f_{1} \equiv 1$ and $f_{2}=1_{T}$. Futher choose $\phi(x)=0$ for $x \neq x_{0}$ and $0<\phi\left(x_{0}\right)<1$.

2.11. Definition. Let $S$ be a subset of $X$. We will say that $S$ is a set of uniqueness (relative to the system $(X, \lambda, F))$ when $\phi=1_{S}$ is a uniqueness function. Equivalently, $M_{0}(S)=\left\{1_{S}\right\}$. Equivalently, no other measure $\psi d \lambda$, 
with $0 \leq \psi \leq 1$, has precisely the same moments as $d \mu_{S}=1_{S} d \lambda$ relative to each $f \in F(S)$, that is, each $f \in F$ such that $\int_{S}|f| d \lambda<\infty$.

We will call $S$ a weak set of uniqueness if $M_{0}(S)$ contains no indicator function $1_{T}$ other than $1_{S}$. Equivalently, no other measure of the special form $d \mu_{T}=1_{T} d \lambda$ has precisely the same moments as $d \mu_{S}=1_{S} d \lambda$ relative to each $f \in F(S)$. Roughly speaking, no other subset $T$ of $X$ has the same $F$-moments as $S$.

As a simple example, choose $X=\{u, v\}$ with $p=\lambda(\{u\})$ and $q=\lambda(\{v\})$, such that $p, q>0$ and $p \neq q$. Let further $F=\{1\}$. Then $S=\{u\}$ is a weak set of uniqueness which is not a set of uniqueness. The latter because there exists a nonindicator function $\phi \in M_{0}$ such that $\phi d \lambda$ has the same mass $p$ as $1_{S} d \lambda$. Namely, define $\phi(u)=1-q / \rho$ and $\phi(v)=p / \rho$, where $\rho \geq \max (p, q)>0$.

2.12. Remarks. In order that the empty set $\varnothing$ be a set of uniqueness, it is necessary and sufficient that there does not exist any nonzero $\phi \in M_{0}$ which is perpendicular to $F$, in the sense that $\int|f| \phi d \lambda<\infty$ and $\int f \phi d \lambda=0$, for all $f \in F$ and, hence, for all $f \in F^{*}=\operatorname{span} F$. It suffices that $F^{*}$ contains a function $f^{+}>0$, (that is, such that $f^{+}(x)>$ a.e. $[\lambda]$ ). Similarly, $X$ itself is set of uniqueness if and only if there exists no nonzero $\phi \in M_{0}$ such that $\int f \phi d \lambda=0$ for all $\lambda$-integrable functions $f \in F$. Hence, in order that $X$ itself be a set of uniqueness, it is at least necessary that the empty set be a set of uniqueness. It would be sufficient that $f^{+}>0$ with $f^{+}$as a linear combination of $\lambda$-integrable functions $f \in F$, (as happens when $1 \in F$ and $\lambda(X)<\infty$ ).

For the moment, suppose that $1 \in F$ and further that each $f \in F$ is $\lambda$ integrable, in particular, $\lambda(X)<\infty$. Choose $\phi \in M_{0}$ and put $\phi_{1}=1-\phi$, thus, $\phi_{1} \in M_{0}$. As is easily seen, $M_{0}\left(\phi_{1}\right)=\left\{1-\psi: \psi \in M_{0}(\phi)\right\}$. Therefore, $\phi$ is a uniqueness function if and only if $\phi_{1}$ is a uniqueness function. Similarly, letting $\phi=1_{S}$, a subset $S$ of $X$ is a (weak) set of uniqueness if and only if its complement $S^{C}=X / S$ is a (weak) set of uniqueness.

In general, the larger the set $F(\phi)$, the easier it is for $\phi$ to be a uniqueness function. At first reading, the reader may prefer to think only of the case $F(\phi)=F$. Namely, by allowing that $F(\phi)$ is a proper subset of $F$, some of the arguments below become more subtle. On the other hand, the assumption that $F(\phi)=F$, for all $\phi \in M_{0}$ (such as $\phi \equiv 1$ ) seems unnecessarily severe, since it is equivalent to the strong assumption that each $f \in F$ be $\lambda$-integrable.

As an illustration, in the present setup, it is possible that $\psi \in M_{0}(\phi)$, (thus $M_{0}(\phi) \supset M_{0}(\psi)$, see 2.9$)$, while simultaneously $\phi \notin M_{0}(\psi)$, thus, $M_{0}(\psi)$ is a proper subset of $M_{0}(\phi)$. This happens precisely when $F(\phi)$ is a proper subset of $F(\psi)$, (and hence of $F$ ). In such a situation, it is even conceivable that $M_{0}(\psi)=\{\psi\}$. Thus, even when $\phi$ itself is not a uniqueness function, it is not excluded that some other $\psi \in M_{0}(\phi)$ happens to be a uniqueness function. Similarly, even when $S$ is not a weak set of uniqueness, it is conceivable that $M_{0}(S)$ contains another indicator function $1_{T}$, such that $T$ is a weak set of uniqueness, (necessarily with $F(T)$ strictly larger than $F(S)$, as would be 
impossible when $F(S)=F$ ). The following result will not be needed futher on but has a clear interest of its own.

2.13. Lemma. Let $S$ be a subset of $X$ and put $G=F(S)$. Then in order that $S$ be a set of uniqueness, it is necessary that the corresponding moment point $c(S)=c_{G}(S)$ be an extreme point of the moment space $C_{G}$, (see (2.4) and $(2.5))$.

2.14. Proof. Let $\phi=1_{S}$ and $G=F(S)$. Suppose that

$$
c(S)=c_{G}(\phi)=\alpha c_{G}\left(\phi_{1}\right)+(1-\alpha) c_{G}\left(\phi_{2}\right), \quad \text { where } 0<\alpha<1,
$$

and $\phi_{1}, \phi_{2} \in M_{0}$ satisfying $F\left(\phi_{1}\right) \cap F\left(\phi_{2}\right) \supset G$. Letting $\psi=\alpha \phi_{1}+(1-\alpha) \phi_{2}$, one has $\psi \in M_{0} ; F(\psi) \supset G$ and $c_{G}(\psi)=c_{G}(\phi)$. But $S$ is a set of uniqueness, hence, $\psi=\phi=1_{S}$ having its values in $\{0,1\}$, therefore, $\phi_{1}=\phi_{2}=1_{S}$ (both a.e. $[\lambda])$, showing that $c_{G}\left(\phi_{1}\right)=c_{G}\left(\phi_{2}\right)$.

By the way, a similar proof shows that $c_{G}(\phi)=\alpha c_{G}\left(\phi_{1}\right)+(1-\alpha) c_{G}\left(\phi_{2}\right)$ with $0<\alpha<1 ; F(\phi) \subset F\left(\phi_{1}\right) \cap F\left(\phi_{2}\right)$ and $\phi$ is a uniqueness function, can only happen when also $\phi_{1}$ and $\phi_{2}$ are uniqueness functions.

2.15. Some natural questions would be as follows.

(i) Exactly when is a given $\phi \in M_{0}$ a uniqueness function? If not, what can be said about the "size" of the set of measures $M(\mu)$, (where $d \mu=\phi d \lambda$ ), such as its total variation diameter?

(ii) Suppose that $1 \in F$. Let $S$ be a subset of $X$ with $\lambda(S)<\infty$, that is, $1 \in F(S)$, and let $\phi=1_{S}$. If $S$ is not a set of uniqueness, one would be interested in the size of the convex set $M(S)$ (of measures $\mu \in M$ having the same $F$-moments as $S$ ). For instance, one would like to know more about the quantity

$$
\varepsilon(S)=\frac{1}{2} \sup \left\{\left\|\nu-\mu_{S}\right\|: \nu \in M(S)\right\}=\frac{1}{2} \sup \left\{\int|\psi-\phi| d \lambda: \psi \in M_{0}(S)\right\} .
$$

Here, $\|\cdot\|$ stands for total variation distance. Since $1 \in F(S)$, each $\psi \in M_{0}(S)$ satisfies $\int(\phi-\psi) d \lambda=0$, hence,

$$
\varepsilon(S)=\sup \left\{\int_{S}(1-\psi) d \lambda: \psi \in M_{0}(S)\right\} .
$$

Note that $\varepsilon(S)=0$ if and only if $S$ is a set of uniqueness. A small value of $\varepsilon(S)$ would indicate that the set $S$, (regarded as a measure $\phi d \lambda$ with $\phi \in M_{0}$ ), is at least nearly determined by its associated moment point $c_{G}(S)$; that is, by the moments $\int f \phi d \lambda=\int_{S} f d \lambda$, one for each $f \in F$ such that $\int_{S}|f| d \lambda<\infty$.

(iii) Let $\mu \in M$, that is, $d \mu=\phi d \lambda$ with $\phi \in M_{0}$. For $\theta: X \rightarrow \mathbf{R}$ as a given (measurable) function, how can one calculate or approximate a quantity of the type

$$
L(\theta)=L(\theta, \mu)=\inf \left\{\int_{X} \theta d \nu: \nu \in M(\mu)\right\} ?
$$

Here, we take $L(\theta)=-\infty$ as soon as $\int \min (0, \theta) d \nu=-\infty$ for some $\nu \in$ $M(\mu)$. 
2.18. As to question (iii), where $d \mu=\phi d \lambda$ is given, note that $\theta \geq f-(f-\theta)_{+}$, (with $z_{+}=\max (z, 0)$ ). Here,

$$
\int f d \nu=\int f d \mu \text { and } \int(f-\theta)_{+} d \nu \leq \int(f-\theta)_{+} d \lambda,
$$

when $f \in F(\phi)^{*}$ and $\nu \in M(\mu)$, (that is, $d \nu=\psi d \lambda$ with $\left.\psi \in M_{0}(\phi)\right)$. It follows that

$$
L(\theta) \geq \sup \left\{\int f d \mu-\int(f-\theta)_{+} d \lambda: f \in F(\phi)^{*}\right\} .
$$

Writing $f=\theta+(f-\theta)_{+}-(\theta-f)_{+}$, this is equivalent to

$$
\begin{aligned}
& \sup \left\{\int \theta(\phi-\psi) d \lambda: \psi \in M_{0}(\phi)\right\}=\int \theta \phi d \lambda-L(\theta) \\
& \quad \leq \inf \left\{\int(\theta-f)_{+} \phi d \lambda+\int(f-\theta)_{+}(1-\phi) d \lambda: f \in F(\phi)^{*}\right\} .
\end{aligned}
$$

Suppose $\phi \in M_{0}$ is of the form $\phi=1_{S}$ and further that $1 \in F$ and $\lambda(S)<\infty$, thus $1 \in F(S)$. Then (2.15) and (2.19), with $\theta=1_{S}$, yield that

$$
\varepsilon(S) \leq \inf \left\{\int_{S}(1-f)_{+} d \lambda+\int_{S^{c}} f_{+} d \lambda: f \in F(S)^{*}\right\},
$$

where $S^{c}=X / S$. Sufficient conditions in order that (2.19), (2.20), (2.21) hold with the equality sign follow from results in $[8,9,10]$. In a subsequent paper, we hope to give a detailed discussion of problems (ii), (iii) as well as concrete applications to the uniqueness problem.

2.22. Optimization. Uniqueness problems of the above type may arise as follows. Let $F$ be any set of functions $f: X \rightarrow \mathbf{R}$, let further $d_{f}(f \in F)$ be given real numbers and consider the convex set

$$
M^{d}=\left\{\psi \in M_{0}: \int|f| \psi d \lambda<\infty ; \int f \psi d \lambda=d_{f}, \text { for all } f \in F\right\} .
$$

We will assume that $M^{d}$ is nonempty. If $M^{d}=\{\phi\}$ then $\phi$ would be uniqueness function relative to $(X, \lambda, F)$, (having $F(\phi)=F)$. One is often interested in

$$
\begin{aligned}
& \rho(h)=\sup \left\{\int h \psi d \lambda: \psi \in M^{d}(h)\right\}, \\
& \text { where } M^{d}(h)=\left\{\psi \in M^{d}: \int|h| \psi d \lambda<\infty\right\},
\end{aligned}
$$

with $h: X \rightarrow \mathbf{R}$ as a given function. Suppose that this supremum is finite and, moreover, is assumed by some $\phi \in M^{d}(h)$; sufficient conditions for this can be found in $[8,9]$. Such functions will be said to be optimal. Let $M^{\#}$ denote the class of all optimal functions. Natural questions would be:

(i) Does $M^{\#}$ contain any indicator function ('bang-bang' control)? 
(ii) Is the optimal $\phi$ unique? If not, how serious is this nonuniqueness? These problems fit in the previous framework, but with $F$ replaced by

$$
F_{h}=F \cup\{h\} .
$$

Let $M_{0, h}(\phi)$ denote the class $M_{0}(\phi)$, as defined in (2.9), but taken relative to $\left(X, \lambda, F_{h}\right)$ instead of $(X, \lambda, F)$. Observe that, for $\phi \in M^{\#}$, all moments $\int f \phi d \lambda\left(f \in F_{h}\right)$ exist and are independent of the particular choice of $\phi$. That is, $F_{h}(\phi)=F_{h}$ and $M_{0, h}(\phi)=M^{\#}$. Equivalently, if $\phi \in M^{\#}$ then

$$
\left\{\psi \in M_{0}: \int|f| \psi d \lambda<\infty ; \int f \psi d \lambda=\int f \phi d \lambda \text {, for all } f \in F_{h}\right\}=M^{\#} .
$$

Let $\phi \in M^{\#}$. Question (i) above thus simply asks whether or not $M_{0, h}(\phi)$ does contain any indicator function. And (ii) above asks whether or not $M_{0, h}(\phi)=$ $\{\phi\}$, that is, whether or not $\phi$ is a uniqueness function, this time relative to the system $\left(X, \lambda, F_{h}\right),($ see 2.10$)$. Problems of this nature are extensively studied in the subsequent sections.

\section{Generalized ADDITIVE SETS}

Here, we use the notations of $\xi 2$. Thus $(X, \lambda)$ is a given $\sigma$-finite measure space and $F$ a given set of functions $f: X \rightarrow \mathbf{R}$. The linear span of a set $H$ of functions is denoted as $H^{*}$. Terms such as 'almost all' or 'a.e.' are always relative to $\lambda$. In the present section, we develop sufficient conditions for $\phi \in M_{0}$ to be a uniqueness function. In the sequel, $f^{+}: X \rightarrow \mathbf{R}$ denotes a variable function such that $f^{+}(x)>0$ on certain subsets of $X$. In many applications, $f^{+}(x) \equiv 1$ is admissible.

3.1. Definition. A (measurable) subset $S$ of $X$ will be said to be an additive set (relative to $F$ or the system $(X, \lambda, F)$ ) if the following hold. Here, as usual, one identifies sets which differ only by a set of $\lambda$-measure zero.

(i) The set $S$ is of the form

$$
\text { either } S=\left\{x \in X: f_{0}(x) \geq 0\right\} \text { or else } S=\left\{x \in X: f_{0}(x)>0\right\} \text {, }
$$

for some $f_{0} \in F(S)^{*}$. Recall that $F(S)^{*}$ is the linear span of $F(S)=\{f \in$ $\left.F: \int_{S}|f| d \lambda<\infty\right\}$. Let $D=\left\{x \in X: f_{0}(x)=0\right\}$.

(ii) There exists $f^{+} \in F(S)^{*}$ such that $f^{+}(x)>0$, for almost [ $\left.\lambda\right]$ all $x \in D$, with $D$ as above. This would be trivially true when $\lambda(D)=0$.

3.3. Remarks. If the set $F$ is linear then condition (i) requires precisely that $S$ be of the form (3.2), with $f_{0} \in F$ such that $\int\left(f_{0}\right)_{+} d \lambda<\infty$, showing that the above definition is not as circular as it may seem at first sight. Here, $z_{+}=$ $\max (0, z)$. In many applications, each $f \in F$ is $\lambda$-integrable and then $F(S)=$ $F$, for all $S$. Often all sets $S$ satisfy (ii), simply because of the existence of a strictly positive $\lambda$-integrable function $f^{+} \in F$, (thus, $f^{+} \in F(S)$, for all $S$ ). For instance, $f^{+}=1$ will do if $\lambda(X)<\infty$ and $1 \in F$. 
For important special cases, an analogous notion, of additive set or function, was already studied by Krein and Nudel'man [14; 15, p. 240], Carrington [2], Fishburn, Lagarias, Reeds, and Shepp [3, 4]. See Kemperman [11, 12] for related results.

The terminology "additive" arises from $F(S)^{*}$ being the linear span of $F(S)$. In many applications, the latter set is quite small, (e.g., finite or countable). Observe that the above additivity implies that $\phi=1_{S}$ attains each of the suprema

$$
\begin{gathered}
\sup \left\{\int f_{0} \psi d \lambda: \psi\right. \\
\left.\in M_{0}(S)\right\}=\sup \left\{\int f_{0} \psi d \lambda: 0 \leq \psi \leq 1\right\} \\
=\int\left(f_{0}\right)_{+} d \lambda=\int_{S} f_{0} d \lambda .
\end{gathered}
$$

Here, $f_{0} \in F(S)^{*}$ is as in condition (i). The following Lemma is a straightforward generalization of some known results, see [3,11].

3.5. Lemma. An additive set $S$ is always a set of uniqueness.

3.6. Proof. Let $S$ be additive as in 3.1 and consider $\psi \in M_{0}(S)$. Thus $0 \leq$ $\psi \leq 1$ and $\int f \psi d \lambda=\int f 1_{S} d \lambda$, each side being finite, for all $f \in F(S)^{*}$. Choosing $f=f_{0}$, one has $\int f_{0}\left(1_{S}-\phi\right) d \lambda=0$. From (3.2), the latter integrand is everywhere nonnegative, implying that $\phi(x)=1_{S}(x)$ a.e. [ $[\lambda]$ on $D^{c}=X / D$, where $D=\left\{x \in X: f_{0}(x)=0\right\}$. We are ready when $\lambda(D)=0$. If not then by (ii) there exists $f^{+} \in F(S)$ such that $f^{+}(x)>0$ a.e. [ [ ] on $D$. Similarly as above, one has $\int f^{+}\left(1_{S}-\phi\right) d \lambda=0$ thus $\int_{D} f^{+}\left(1_{S}-\phi\right) d \lambda=0$. In view of (3.2), the latter integrand has a constant sign showing that $\phi(x)=1_{S}(x)$ a.e. $[\lambda]$ also on $D$.

3.7. Remarks. It is clear from the proof that an additive set $S$, depending on $f_{0}, f^{+} \in F(S)^{*}$ as in 3.1 , is already a set of uniqueness relative to $(X, \lambda, H)$ with $H$ as the tiny set $H=\left\{f_{0}, f^{+}\right\}$, (often with $f^{+}=1$ ).

Suppose $S$ is of the form (3.2) with $f_{0} \in F(S)^{*}$ and, moreover, that $1 \in F$ and $\lambda(S)<\infty$, thus $1 \in F(S)$. In this case, another proof would be to show that the right-hand side of (2.21) equals zero. In the second case (3.2), this follows by choosing $f=\rho f_{0}$ with $\rho>0$ large. Then $f \in F(S)^{*}$ while $f \leq 0$ on $S^{c}$, hence, the second integral (2.21) equals zero. Further, $f_{0}>0$ on $S$, hence, $\left(1-\rho f_{0}\right)_{+} \rightarrow 0$ on $S$, as $\rho \rightarrow \infty$, hence, the first integral (2.21) tends to zero (by the bounded convergence theorem, since $\lambda(S)<\infty$ ). At least when $\lambda$ is finite, (thus, $\lambda\left(S^{c}\right)<\infty$ ), a similar proof applies to the first case (3.2), this time choosing $f=1+\rho f_{0}$ with $\rho>0$ large.

3.8. Illustration. Take $X=\mathbf{R}$ and let $\lambda$ be Lebesgue measure on $\mathbf{R}$. Let $m$ be a fixed positive integer and choose $F=P_{2 m}$ as the linear set of all (real-valued) polynomials $f$ on $\mathbf{R}$ of degree $\leq 2 \mathrm{~m}$. Let $\Sigma_{2 m}$ denote the collection of all subsets $S$ of $\mathbf{R}$ which are unions of at most $m$ finite intervals, including the empty set. 
If $S$ contains an infinite interval (such as $S=X$ ) then $F(S)=\{0\}$, thus $M_{0}(S)=M_{0}$, showing that $S$ cannot possibly be a set of uniqueness, hence, $S$ is not additive either. Using this remark, it is easily seen that a subset $S$ of $\mathbf{R}$ is additive if and only if it is a bounded set of the form $S=\left\{x \in X: f_{0}(x) \geq 0\right\}$, with $f_{0}$ as a nonzero polynomial of degree $\leq 2 \mathrm{~m}$; (and one can choose $f^{+}=1$ in Definition 3.1). Equivalently, a subset $S$ of $\mathbf{R}$ is additive if and only if $S \in \Sigma_{2 m}$. Hence, from Lemma 3.5, each $S \in \Sigma_{2 m}$ is a set of uniqueness. For each such set $S$, one has $\phi=1_{S}$ a.e. as soon as $0 \leq \phi \leq 1$ and

$$
\int x^{j} \phi(x) d x=\int_{S} x^{j} d x \text { for all } 0 \leq j \leq 2 m ;
$$

(or all $0 \leq j \leq 2 k$ if $S$ is the union of $k \leq m$ intervals). Conversely, it is also true that any uniqueness function $0 \leq \phi \leq 1$ must be of the form $\phi=1_{S}$ with $S \in \Sigma_{2 m}$. This assertion is a consequence of results in Krein and Nudel'man [15, pp. 238-242], Karlin and Studden [7, p. 235], and also follows from much more general results in $\S 4$, see Remark 4.13.

3.10. Definition. Let $\phi \in M_{0}$ and put $G=F(\phi)$. Let $s$ be a nonnegative integer. We will say that $\phi$ is $s$-additive if, for some choice of $f_{r} \in F^{*} \quad(r=$ $0, \ldots, s)$, the following properties (i), (ii) are true. Here,

$$
\begin{aligned}
& S=\left\{x \in X: f_{q}(x)=0 \text { for } 0 \leq q<r ; f_{r}(x)>0 ; \text { for some } r=0, \ldots, s\right\} ; \\
& T=\left\{x \in X: f_{q}(x)=0 \text { for } 0 \leq q<r ; f_{r}(x)<0 ; \text { for some } r=0, \ldots, s\right\} ; \\
& Z=\left\{x \in X: f_{r}(x)=0 \text { for all } 0 \leq r \leq s\right\} .
\end{aligned}
$$

Thus, $S, T$, and $Z$ are disjoint with union $X$.

(i) We have $\phi(x)=1$ for almost all $x \in S$ and $\phi(x)=0$ for almost all $x \in T$.

(ii) Moreover, $G=F(\phi)$ is a $Z$-determining set (of functions). Which is the same as $G^{*}$ being $Z$-determining. The present condition (ii) is trivially satisfied when $\lambda(Z)=0$.

In general, if $H$ is any set of functions $f: X \rightarrow \mathbf{R}$ and $Z$ a subset of $X$, we will say that $H$ is $Z$-determining if a function $\psi \in M_{0}[H]$, which is supported by $Z$, is completely determined by the set of moments $\int f \psi d \lambda$ $(f \in H)$. Equivalently, $\psi(x)=0$ a.e. [ $\lambda]$ as soon as $\psi: X \rightarrow \mathbf{R}$ is a bounded function satisfying

$$
\psi(x)=0 \text { for } x \notin Z ; \int|f| \psi d \lambda<\infty, \int f \psi d \lambda=0, \quad \text { for all } f \in H .
$$

3.12. In the special case that $G$ is $X$-determining we will also regard each $\phi \in M_{0}$ to be $s$-additive with $s=-1$. In this case, choosing $s=0$ and $f_{0}=0 \in F(S)^{*}$, the function $\phi$ is also 0 -additive. In general, $s$-additivity implies $(s+1)$-additivity, as can be seen by choosing $f_{s+1}=f_{s}$. 
A subset $S$ of $X$ is said to be an $s$-additive if its indicator function $\phi=1_{S}$ is $s$-additive. The associated integer $s$ and functions $f_{0}, \ldots, f_{s} \in F(S)^{*}$ need not be unique. We will say that the set $S$ is a generalized additive set if it is $s$ additive for some integer $s \geq-1$. Similarly for generalized additive functions.

3.13. Of special interest is the case $\lambda(Z)=0$. Here, condition (ii) above is trivially satisfied while $\phi=1_{S}$ is an indicator function. An $s$-additive set, such that $\lambda(Z)=0$ for the associated set $Z$, will be called a special s-additive set (or also a special generalized additive set). The situation $\lambda(Z)=0$ occurs in 3.10 if and only if the functions $f_{0}, \ldots, f_{s} \in F(\phi)^{*}$ are such that $f_{r}(x)=0$ for all $0 \leq r<s$ implies that $f_{s}(x) \neq 0$, (a.e. $\left.[\lambda]\right)$.

In particular, each additive set is a special 1-additive set. Namely, let $f_{0}$, $f^{+} \in F(S)$ be as in Definition 3.1 and choose $s=1$ and $f_{1}=c f^{+}$in 3.10, $c$ as a nonzero constant. This leads to the additive set $S=\left\{f_{0}(x) \geq 0\right\}$ or $S=$ $\left\{f_{0}(x)>0\right\}$, respectively, depending on whether $c>0$ or $c<0$, respectively. If $\lambda\left(\left\{f_{0}(x)=0\right\}\right)=0$ then the additive set $S$ on hand would even be 0 additive. As to the converse, there are examples (see 3.22 and 6.35) of special 1-additive sets $S$ which are not additive. In such a situation

$$
\begin{aligned}
S=\left\{x \in X: f_{0}(x)>0 \text { or } f_{0}(x)=0 ; f_{1}(x)>0\right\}, & \\
& \text { where } \lambda\left(\left\{x \in X: f_{0}(x)=f_{1}(x)=0\right\}\right),
\end{aligned}
$$

$\left(f_{0}, f_{1} \in F(S)^{*}\right)$. Moreover, $D=\left\{x \in X: f_{0}(x)=0\right\}$ satisfies $\lambda(D)>0$ and $f_{1}(x)$ must take both signs on $D$.

3.14. Remark. In certain important applications, the class $F$ is determined by a given collection of mappings $\pi_{j}: X \rightarrow Y_{j}(j \in J)$. Typically, $F$ is then chosen as the (nonlinear) set of all functions $f: X \rightarrow \mathbf{R}$ of the special form $f(x)=g_{j}\left(\pi_{j} x\right)$, where $j \in J$ and $g_{j}: Y_{j} \rightarrow \mathbf{R}$, see $\S 6$ for details. In the papers $[3,4,5,11]$ it is further assumed that $\lambda(X)<\infty$, thus, $1 \in F(S)$, while there a set $S$ was said to be additive if it is of the form $S=\left\{x \in X: f_{0}(x) \geq 0\right\}$, for some $f_{0} \in F(S)^{*}$. In [11] we introduced the notion of a set $S$ being additive of degree $\leq s$. This means that $f_{r} \in F(S)^{*} \quad(r=0, \ldots, s)$ can be found, such that $x \in S$ if and only if, either $f_{r}(x)=0(r=0, \ldots, s)$ or else there exists $0 \leq r \leq s$ such that $f_{q}(x)=0$ for $0 \leq q<r$ and $f_{r}(x)>0$. Choosing $f_{s+1}=1 \in F(S)$, we see the additivity of degree $\leq s$ in the sense of [11] implies $(s+1)$-additivity in the present sense (with the associated set $Z$ as the empty set).

3.15. Remark. Let $G=F(\phi)$ and $Z$ be as in 3.10, with $G$ as a $Z$-determining set. Consider for the moment the case that the dimension $k$ of $\left\{f_{Z}: f \in\right.$ $\left.G^{*}\right\}$ is finite, (with $f_{Z}$ as the restriction of $f: X \rightarrow \mathbf{R}$ to $Z$ ). Then, as is easily seen, $G$ being $Z$-determining means precisely that the measure $\lambda$ restricted to $Z$ determines a measure space consisting of precisely $k$ atoms, to the effect that each (measurable) function on $Z$ is the restriction $f_{Z}$ of some $f \in G^{*}$. For instance, $k=0$ if and only if $\lambda(Z)=0$. Moreover, $0<k<\infty$ implies 
that $(X, \lambda)$ must have atoms. In particular, if $(X, \lambda)$ is nonatomic and $F$ is finite then a generalized additive function $\phi$ must be special. That is, one must have $\lambda(Z)=0$ for the associated set $Z$, implying that $\phi$ is an indicator function. This would also follow from Theorem 3.17 and Lemma 4.4.

3.16. Remark. For the moment, suppose that each $f \in F$ is $\lambda$-integrable. Thus, $F(\psi)=F$ for all $0 \leq \psi \leq 1$. Choose $f_{r} \in F^{*}(r=0, \ldots, s)$ such that the associated set $Z$ as in (3.11) has positive $\lambda$-measure. Suppose further that $F$ is $Z$-determining, as happens when sufficiently many functions supported by $Z$ are equal to the restriction of some $f \in F^{*}$. In such a situation, $\psi: X \rightarrow$ $[0,1]$ is $s$-additive as soon as $\psi(x)=1 \quad(x \in S)$ and $\psi(x)=0 \quad(x \in T)$, whatever the values $0 \leq \psi(x) \leq 1 \quad(x \in Z)$. Here, $S$ and $T$ are defined by (3.11). In view of the following Theorem 3.14, any such $\psi$ is also a uniqueness function. However, most of these functions $\psi$ are not indicator functions.

As a trivial example, if $X=\left\{x_{0}\right\}$ and $F=\{1\}$ then $F$ is $X$-determining so that each $\phi \in M_{0}$ is $s$-additive with $s=-1$. Choosing $s=0$ and $f_{0}=0 \in F^{*}$, we see that each $\phi \in M_{0}$ is also 0 -additive with associated set $Z=X$.

As another illustration, let $X=\mathbf{R}$ with Lebesgue measure $\lambda$. Let further $F$ consist of all functions $f: X \rightarrow \mathbf{R}$ that are linear for $x \geq 0$, and arbitrary for $x<0$. Then, with $c$ as any positive constant, $\phi \in M_{0}$ is 0 -additive (and thus is a uniqueness function) as soon as $\phi(x)=1$ for $0 \leq x \leq c$ and $\phi(x)=0$ for $x>c$, whatever the values $0 \leq \phi(x) \leq 1$ with $x<0$. After all, choose $s=0$; $f_{0}(x)=0$ for $x<0$ and $f_{0}(x)=c-x$ for $x \geq 0$, thus, $Z=(-\infty, 0)$. It is obvious that $F(\phi)$ is $Z$-determining.

3.17. Theorem. In order that $\phi \in M_{0}$ be a uniqueness function it is sufficient that $\phi$ be a generalized additive function. Similarly, a generalized additive set $S$ is always a set of uniqueness.

3.18. Proof. Let $\phi \in M_{0}$ be $s$-additive as described in 3.10, involving functions $f_{r} \in G^{*} \quad(r=0, \ldots, s)$, where $G=F(\phi)$. The case $s=-1$ is trivial, (for, then each $\phi \in M_{0}$ is a uniqueness function), thus, let $s \geq 0$. Let $\psi \in M_{0}(\phi)$ be fixed, thus, $0 \leq \psi \leq 1$ and further

$$
\int|f| \psi d \lambda<\infty \text { and } \int f(\psi-\phi) d \lambda=0, \quad \text { for all } f \in G^{*} .
$$

In particular since $f_{r} \in G^{*}$,

$$
\int f_{r}(x)(\phi(x)-\psi(x)) \lambda(d x)=0
$$

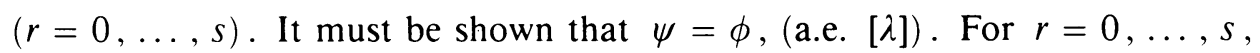
put

$$
\begin{aligned}
& Q_{r}=\left\{x \in X: f_{q}(x)=0 \text { for } 0 \leq q<r ; f_{r}(x) \neq 0\right\} ; \\
& Z_{r}=\left\{x \in X: f_{q}(x)=0 \text { for } 0 \leq q \leq r\right\} .
\end{aligned}
$$

Note that $X$ is the disjoint union of $Q_{0}, \ldots, Q_{s}$ and $Z_{s}=Z$. From 3.10, if $x \in Q_{r}$ then $\phi(x)=1$ or 0 depending on whether $f_{r}(x)>0$ or $f_{r}(x)<0$, 
respectively. Thus, the integrand in (3.20) is nonnegative on $Q_{r}$ and equals zero on $Q_{r+1} \cup \cdots \cup Q_{s} \cup Z=Z_{r},(r=0, \ldots, s)$. Taking $r=0$, it follows that $\psi-\phi=0$ on $Q_{0}$. Afterwards, taking $r=1$, one obtains that $\psi-\phi=0$ on $Q_{1}$ and so on. In this way, we see that $\psi-\phi=0$ on $Q_{0} \cup \cdots \cup Q_{s}=Z^{c}$ and it only remains to show that $\psi-\phi=0$ also on $Z$. But this follows immediately from (3.19) and the fact that $G^{*}$ is $Z$-determining, (see 3.10).

3.21. Remark. Suppose that $S$ is a special generalized additive set. Thus, $s \geq 0$ and $f_{0}, \ldots, f_{s} \in F(S)^{*}$ can be found such that $S$ is of the form (3.11), while, simultaneously, $\lambda(Z)=0$ for the associated set $Z$. It follows that $S$ is a special generalized additive set (and, thus, a set of uniqueness) also relative to the usually much smaller system $(X, \lambda, H)$ with $H=\left\{f_{0}, \ldots, f_{s}\right\}$. In particular, $\phi=1_{S}$ as soon as $\phi \in M_{0}$ satisfies $\int\left|f_{r}\right| \phi d \lambda<\infty$ and $\int f_{r} \phi d \lambda=$ $\int_{S} f_{r} d \lambda(r=0, \ldots, s)$.

3.22. Illustration. The following is an example of a set $S$ of uniqueness which is not additive (as defined in 3.1; see 6.35 for a related result). In fact, $S$ will be chosen as a special 1-additive set of the form

$$
S=\left\{x \in X: f_{0}(x)>0\right\} \cup\left\{x \in X: f_{0}(x)=0 ; f_{1}(x)>0\right\} .
$$

Here, $f_{0}, f_{1} \in F(S)$ will be such that $Z=\left\{x \in X: f_{0}(x)=f_{1}(x)=0\right\}$ satisfies $\lambda(Z)=0$. Namely, choose $X=(-1,+\infty)$ with Lebesgue measure $\lambda$. Let further $F$ be the linear span of the two functions $u_{1}(x)=x(1-x)$; $u_{2}(x)=x^{2}$ if $x \leq 0$ and $u_{2}(x)=0$ if $x \geq 0$. Now choose $f_{0}=u_{2}$ and $f_{1}=u_{1}$. Then $S$ as defined by (3.23) takes the form $S=(-1,+1)$ while the associated set $Z=\{0,1\}$ indeed satisfies $\lambda(Z)=0$. Each $u_{i}$ is integrable on $S$ thus $F(S)=F$. It follows that $S=(-1,+1)$ is 1 -additive, hence, $S$ is a set of uniqueness. This presently means that $\phi=1_{S}$ is the only function $0 \leq \phi \leq 1$ on $(-1, \infty)$ which satisfies

$$
\int_{-1}^{\infty} x(1-x) \phi(x) d x=-2 / 3 ; \quad \int_{-1}^{0} x^{2} \phi(x) d x=1 / 3 .
$$

We claim that $S$ is not additive (relative to $(X, \lambda, F)$ ). For, otherwise, either $(-1,+1)=\{x \in X: g(x)>0\}$ or else $(-1,+1)=\{x \in X: g(x) \geq 0\}$ (up to a set of measure zero), where $g: X \rightarrow \mathbf{R}$ is of the form $g(x)=b x(1-x)+c u_{2}(x)$, with $b$ and $c$ as constants. But $u_{2}(x)=0$ for $x \geq 0$, while $g$ must change sign at $x=1$, hence, $b>0$. Since $u_{2}(x)=x^{2}=o(x)$, for $x \leq 0$, it would follow that, for $x<0$ small, $g(x) \approx b x$, thus, $g(x)<0$ and we have a contradiction. This construction is easily modified so as to yield, for any $s$, an example of an $s$-additive set which is not $(s-1)$-additive.

3.24. Remarks. By the way, it follows from the results in $\S 4$, see also 1.13 , that in the present example every set $S$ of uniqueness must be of the form (3.23) with $f_{0}, f_{1} \in F(S)$ such that $f_{0} \neq 0$ and $\lambda(Z)=0$, (possibly $f_{1}=\gamma f_{0}$ ). The empty set $S=\varnothing$ is presently not a set of uniqueness, since $T=(0,3 / 2)$ has the same $F$-moments as $S$. 
The set $S=(-1,+1)$ remains a nonadditive set of uniqueness even when one replaces $F$ by the linear span of $f^{+}=1, u_{1}$, and $u_{2}$. Note that the nonadditive set of uniqueness $S$ is of the limit of the additive set

$$
S_{\rho}=(-1,-\rho) \cup(0,1)=\left\{x \in X: \rho u_{1}(x)+(1-\rho) u_{2}(x)>0\right\} \quad \text { as } \rho \downarrow 0 .
$$

As a related feature, it is even possible that a sequence $\left\{S_{n}\right\}$ of additive sets converges to a limiting set $S$ which is not even a set of uniqueness. Namely, Fishburn et al. [3] showed that this can happen for the subset $X=[0,1]^{3}$ of $\mathbf{R}^{3}$ with Lebesgue measure $\lambda$, while $F$ consists (say) of all bounded functions of the form $f(x)=\sum_{j} f_{j}\left(x_{j}\right)$. In fact, for this choice of $(X, \lambda, F)$, it was shown that the set $S=\left\{x \in X: x_{3} \geq \max \left(x_{1}, x_{2}\right)\right\}$ is not a set of uniqueness, though $S$ is the limit of $\left\{x \in X: x_{3}^{n}-x_{1}^{n}-x_{2}^{n} \geq 0\right\}$ as $n \rightarrow \infty$.

\section{The FINITE DIMENSIONAL CASE}

As usual, we identify functions on $X$ which are equal a.e., (relative to $\lambda$ ). It is useful to visualize the $\sigma$-finite measure space $(X, \lambda)$ as being decomposed into a discrete part (determined by a finite or countable number of atoms) and a nonatomic part. Either part could be empty.

If $H$ is any set of functions on $X$ and $Z$ is a subset of $X$, then $\operatorname{dim}(H \mid Z)$ will denote the dimension of the linear span of $\left\{h_{z}: h \in H\right\}$, with $h_{Z}$ as the restriction of $h$ to $Z$. We also write $\operatorname{dim} H=\operatorname{dim}(H \mid X)$, thus, $\operatorname{dim} H=$ $\operatorname{dim} H^{*}$. Let further $N=N(\lambda \mid Z) \leq+\infty$ denote the largest number of disjoint subsets of $Z$, each of positive $\lambda$-measure. Thus, $N=0$ if and only if $\lambda(Z)=$ 0 , while $1 \leq N<\infty$ if and only if $(Z, \lambda)$ is purely atomic with precisely $N$ atoms. In all other cases $N=\infty$. Clearly, $\operatorname{dim}(H \mid Z) \leq N(\lambda \mid Z) \leq \infty$, for all $H$.

4.1. Throughout $\S 4$, we restrict ourselves to the case that $F$ if finite. Presently, letting $m=\operatorname{dim} F=\operatorname{dim}=F^{*}$, the span $F^{*}$ of $F$ is of the form

$$
F^{*}=\left\{f=\alpha_{1} u_{1}(x)+\ldots+\alpha_{m} u_{m}(x) ; \alpha_{1}, \ldots, \alpha_{m} \in \mathbf{R}\right\},
$$

with $u_{i} \in F$ and the $u_{i}: X \rightarrow \mathbf{R}$ as ( $\lambda$-essentially) linearly independent functions. What really matters is the condition $\operatorname{dim} F^{*}<\infty$, and not the finiteness of $F$ itself. On the other hand, one cannot simply replace $F$ by $F^{*}=\operatorname{span} F$, since this may lead to an essentially differen notion of uniqueness function, see the discussion in 2.6.

Let $\phi \in M_{0}$ be fixed. We like to know exactly when $\phi$ is a uniqueness function. Put $G=F(\phi)$, thus, $G$ is the set of all $f \in F$ that are integrable relative to $d \mu=\phi d \lambda$. Letting $k=\operatorname{dim} G$, one may as well assume that $k \geq 1$ since, otherwise, $M_{0}(\phi)=M_{0}$ and $\phi$ could not possibly be a uniqueness function. Let $v_{i} \in G(i=1, \ldots, k)$ be such that $\left\{v_{1}, \ldots, v_{k}\right\}$ is a basis for $G^{*}=\operatorname{span} G$, thus, $1 \leq k \leq m$. Presently, $M_{0}[G]$ as defined by $(2.3)$ coincides with the set of all functions $0 \leq \psi \leq 1$ such that $\int\left|v_{i}\right| \psi d \lambda<\infty, \quad(i=$ $1, \ldots, k)$. Since $G^{*}$ is spanned by $\left\{v_{1}, \ldots, v_{k}\right\}$, the moment point $c_{G}(\psi)$ 
corresponding to $\psi \in M_{0}[G]$ is fully determined by the 'reduced' moment point (4.3)

$$
y(\psi)=\left(y_{1}(\psi), \ldots, y_{k}(\psi)\right) \in \mathbf{R}^{k}, \quad \text { where } y_{i}(\psi)=\int v_{i}(x) \psi(x) \lambda(d x),
$$

$(i=1, \ldots, k)$. Note that the map $y: M_{0}[G] \rightarrow \mathbf{R}^{k}$ is linear. We also write $y(T)=y\left(1_{T}\right)$ with $T$ as a subset of $X$ such that $1_{T} \in M_{0}[G]$.

From the Definition 2.10, $\phi$ is a uniqueness function if and only if there does not exist any $\psi \in M_{0}[G]$ distinct from $\phi$ such that $y(\psi)=y(\phi)$, that is, $y_{i}(\psi)=y_{i}(\phi) \quad(i=1, \ldots, k)$. A subset $S$ of $X$ is said to be a set of uniqueness when $\phi=1_{S}$ is a uniqueness function.

The following result is implicit in the literature, see [7, p. 265; 15, pp. 239242, and 13]. For more general results see Theorems 4.16 and 5.9 below as well as remarks in 5.2 and 5.8 .

4.4. Lemma. Suppose that $(X, \lambda)$ is nonatomic and further that $F^{*}$ is spanned by finitely many functions. Then each uniqueness function is necessarily an indicator function.

4.5. Proof. Let $\phi \in M_{0}$ be a uniqueness function and let $v_{i} \in G=F(\phi) \quad(i=$ $1, \ldots, k)$ define a basis for $G^{*}$, in particular, each $v_{i}$ is integrable relative to $\phi d \lambda$. Suppose first that each $v_{i}$ is $\lambda$-integrable. From Lyapunov's theorem (see Karlin [7, p. 266]), applied to the finite set of measures $\left\{v_{i} d \lambda ; i=1, \ldots, k\right\}$, there exists a subset $S$ of $X$ such that $y_{i}\left(1_{S}\right)=y_{i}(\phi) \quad(i=1, \ldots, k)$, see (4.3). Since $\phi$ is a uniqueness function, it follows that $\phi=1_{s}$.

If some $v_{i}$ is not $\lambda$-integrable then first decompose $X$ into countably many disjoint sets $X_{r}$, such that $v_{1}, \ldots, v_{k}$ are bounded on $X_{r}$ and $0<\lambda\left(X_{r}\right)<\infty$, for all $r$. Let $\phi_{r}, \lambda_{r}$, and $F_{r}$, respectively, be the restrictions to $X_{r}$ of $\phi, \lambda$, and $F$, respectively. Since $\phi$ is a uniqueness function relative to $(X, \lambda, F)$, it is easily seen that $\phi_{r}$ is a uniqueness function relative to $\left(X_{r}, \lambda_{r}, F_{r}\right)$. From the result just obtained, since $\left(X_{r}, \lambda_{r}\right)$ is also nonatomic, it follows, for all $r$, that $\phi_{r}$ is the indicator function of a subset $S_{r}$ of $X_{r}$. Hence, $\phi$ itself is the indicator function of $S=\bigcup S_{r}$.

4.6. With the above notations, let

$$
Y=Y_{G}=\left\{y(\psi): \psi \in M_{0}[G]\right\}
$$

be the set of all reduced moment points, as in (4.3). We call $Y$ the reduced moment space for $G=F(\phi)$. It depends on $\phi$ and the above particular choice of the basis elements $y_{i} \in G$. Clearly, $Y$ is a convex subset of $\mathbf{R}^{k}$ containing the origin $y(0)=0$. The boundary of $Y$ will be denoted as $\partial Y$ and its interior as $\operatorname{int}(Y)$. We assert that $\operatorname{int}(Y)$ is nonempty.

For, otherwise, the convex set $Y$ would be a subset of some hyperplane $\sum_{i} \alpha_{i} y_{i}=0$, (not all $\alpha_{i}$ equal to zero). Equivalently, $v=\sum_{i} \alpha_{i} v_{i}$ is a nonzero function on $X$ satisfying $\int v \psi d \lambda=0$ for all $\psi \in M_{0}[G]$. But this is impossible since $M_{0}[G]$ is quite large. After all, $X$ can be decomposed into countably 
many sets $X_{r}$ with $\lambda\left(X_{r}\right)<\infty$ and such that $v_{1}, \ldots, v_{k}$ are bounded on each $X_{r}$. Hence, $M_{0}[G]$ contains all functions $0 \leq \psi \leq 1$ which are supported by any such set $X_{r}$, (that is, $\psi(x)=0$ for $x \notin X_{r}$ ), and it would follow that $v$ vanishes on each $X_{r}$.

From Lemma 2.13, if $\phi=1_{S}$ with $S$ as a set of uniqueness then $y(\phi)$ is an extreme point of $Y$, hence, $y(\phi) \notin \operatorname{int}(Y)$. A related result is as follows.

4.8. Theorem. Let $\phi \in M_{0}$ be a uniqueness function with associated reduced moment point $y(\phi) \in Y$. Here, $Y=Y_{G}$ is the moment space defined by (4.7). Then either $y(\phi) \in \partial Y$ or else

$$
N(\lambda \mid X)=\operatorname{dim} G=\operatorname{dim} F=m \quad(1 \leq m<\infty) .
$$

In the latter case, the measure space $(X, \lambda)$ is discrete with precisely $m$ atoms while $G^{*}=F^{*}$ consists of all functions $f: X \rightarrow \mathbf{R}$. This in turn implies that each function $0 \leq \psi \leq 1$ on $X$ is a uniqueness function. In particular there exist uniqueness functions $\phi$ which are not indicator functions and even such that $y(\phi) \in \operatorname{int}(Y)$.

4.10. Proof. Clearly, (4.9) implies the subsequent assertions. Suppose $\phi$ is a uniqueness function such that $y(\phi) \notin \partial Y$, that is, $y(\phi) \in \operatorname{int}(Y)$. Since

$$
\operatorname{dim} G \leq \operatorname{dim} F=m \leq N(\lambda \mid X),
$$

it suffices to show that $N(\lambda \mid X) \leq \operatorname{dim} G$.

On the contrary, suppose that $N(\lambda \mid X)>\operatorname{dim} G=k$. That is, there exist $k+1$ disjoint sets $A_{0}, \cdots, A_{k}$ in $X$ with $0<\lambda\left(A_{r}\right)<\infty \quad(r=0, \ldots, k)$. One may even require that each function $v_{i}$ be bounded on each $A_{r} \quad(i=1, \ldots, k$ and $r=0, \ldots, k)$. Therefore, $M_{0}[G]$ contains each function $0 \leq \psi \leq 1$ which is supported by $A=A_{0} \cup A_{1} \cup \cdots \cup A_{k}$. For example, one may choose $\psi(x)=z_{r}=$ constant when $x \in A_{r}\left(r=0, \ldots, k ; 0 \leq z_{r} \leq 1\right)$ and $\psi(x)=0$ if $x \notin A$. There even exist distinct functions $\psi_{1}, \psi_{2} \in M_{0}[G]$ of this type satisfying $y\left(\psi_{1}\right)=y\left(\psi_{2}\right)$, see (4.3). For instance, let $\psi_{1}$ correspond to $z_{r}=\frac{1}{2}$ and $\psi_{2}$ to $z_{r}=\left(1+\varepsilon w_{r}\right) / 2$, where $\varepsilon>0$ is sufficiently small, while the $w_{r}(r=0, \ldots, k)$ are chosen as numbers not all zero satisfy $\sum_{r} a_{i r} w_{r}=0$ $(i=1, \ldots, k)$. Here, $a_{i r}=\int_{A_{r}} v_{i} d \lambda$.

Let $y^{*}=y\left(\psi_{1}\right)=y\left(\psi_{2}\right)$. Since $y^{*} \in Y$ and $y(\phi) \in \operatorname{int}(Y)$, there exists $y\left(\psi^{\#}\right) \in Y$, (where $\left.\psi^{\#} \in M_{0}[G]\right)$, such that $y(\phi)$ belongs to the line segment $\left(y^{*}, y\left(\psi^{*}\right)\right]$. Thus, for some constant $0<\alpha \leq 1$,

$$
\begin{aligned}
y(\phi) & =\alpha y^{*}+(1-\alpha) y\left(\psi^{\#}\right) \\
& =\alpha y\left(\psi_{i}\right)+(1-\alpha) y\left(\psi^{\#}\right)=y\left(\alpha \psi_{i}+(1-\alpha) \psi^{\#}\right),
\end{aligned}
$$

$(i=1,2)$. Since $\phi$ is a uniqueness function, this would imply that $\phi=$ $\alpha \psi_{i}+(1-\alpha) \psi^{\#}(i=1,2)$ thus $\psi_{1}=\psi_{2}$ and we have a contradiction.

4.11. Theorem. Assume that $(X, \lambda)$ is nonatomic, that $\operatorname{dim} F<\infty$ and, $f$ nally, that

$$
\lambda(\{x \in X: f(x)=0\})=0 \text { for each nonzero } f \in F^{*} .
$$


Assertion. A function $\phi \in M_{0}$ is a uniqueness function if and only if it is of the form $\phi=1$ s with $S$ as an additive set (relative to $F$ ). In fact, $S=\{x \in$ $\left.X: f_{0}(x)>0\right\}$ for some nonzero member $f_{0}$ of $F(S) *$.

4.13. Remark. In particular, under the assumptions of Theorem 4.11, the empty set $\varnothing$ is a set of uniqueness (with $F(\varnothing)=F$ ) if and only if $f^{+}(x)>0$ a.e. [ $\lambda$ ] on $X$ for some $f^{+} \in F^{*}$. And $S=X$ is a set of uniqueness if and only if there exists a $\lambda$-integrable function $f^{+}$of this type.

Let us discuss here the possibility of dropping the condition in Theorem 4.11 that $(X, \lambda)$ be nonatomic. One may as well assume that $F$ is nontrivial, (for, otherwise, there does not exist any uniqueness function). Let $x_{0}$ be an atom of $(X, \lambda)$ thus $\lambda\left(\left\{x_{0}\right\}\right)>0$. It is easily seen that (4.12) holds if and only if $F^{*}$ consists of all multiples of a single a.e. nonzero function $g$. Note that $\int g \phi d \lambda\left(\phi \in M_{0}\right)$ takes all values in $[-a, b]$, where $a=\int g_{-} d \lambda \leq \infty$ and $b=\int g_{+} d \lambda \leq \infty$, thus $a+b>0$. There exist no uniqueness functions at all when $a=b=\infty$. The set $S=\{x \in X: g(x)<0\}$ is a set of uniqueness if and only if $a<\infty$. The set $T=\{x \in X: g(x)>0\}$ is a set of uniqueness if and only if $b<\infty$. Besides $1_{S}$ and $1_{T}$ no other uniqueness functions can exist unless $X=\left\{x_{0}\right\}$.

The above argument shows that in Theorem 4.11 the condition that $(X, \lambda)$ be nonatomic can be omitted, provided we rule out the possibility that $(X, \lambda)$ is atomic with a single atom $x_{0}$, while simultaneously $F^{*}$ consists of all the constant functions on $X$. In the latter case, each $\phi \in M_{0}$ is a uniqueness function but it need not be an indicator function.

Theorem 4.11 is also a consequence of Theorem 4.16 below, since under the above assumptions each generalized additive set is already additive, (as described in Theorem 4.11). To see this, first observe that 3.10 can only hold with $\lambda(Z)=0$, since $(X, \lambda)$ is nonatomic and $\operatorname{dim} F<\infty$. Letting $s$ in 3.10 be minimal, we see from (4.12) that necessarily $s=0$, while $f_{0} \in F(S)^{*}$ must be nonzero, showing that $S$ is additive. By the way, the case $s=-1$ cannot arise because $F(S)$ cannot possibly be $X$-determining, since $\operatorname{dim} F(S)<\infty$ and $(X, \lambda)$ is nonatomic.

In many applications, $X$ is an open subset of $\mathbf{R}^{n}$ with $n$-dimensional Lebesgue measure $\lambda$ and then condition (4.12) is satisfied, for instance, when each $f \in F$ is analytic on $X$. Essentially known, see $[1,7,14,15]$, is the special case of Theorem 4.11 that $X=(a, b)$ is a finite or infinite open interval in $\mathbf{R}$ with Lebesgue measure $\lambda$ while $F^{*}$ is spanned by a Tchebycheff system of order $n$. In this situation, (4.12) is always satisfied, hence, it follows from Theorem 4.11 that any set of uniqueness is a union of at most $(n+1) / 2$ intervals. As a special case, taking $X=\mathbf{R}$ and $F=P_{2 m}$ as the polynomials of degree $\leq 2 m$, one obtains the yet unproved assertions in 3.28.

4.14. Proof of Theorem 4.11. If $S$ is additive then $1_{S}$ is a uniqueness function by Lemma 3.25. Conversely, let $\phi \in M_{0}$ be a uniqueness function with associated reduced moment point $y^{0}=y(\phi) \in Y$. Let $k=\operatorname{dim} G$ and the 
$v_{i}$ are as in 4.1. Here, $k \geq 1$, for, otherwise, $\phi$ could not be a uniqueness function.

From Theorem 4.8, since $(X, \lambda)$ has no atoms, we have $y^{0} \in \partial Y$. Since $\operatorname{int}(Y)$ is nonempty, there exist numbers $\alpha_{i}(i=1, \ldots, k)$ not all zero, such that $\sum_{i} \alpha_{i}\left(y_{i}-y_{i}(\phi)\right) \leq 0$, for all $y \in Y$. Equivalently by (4.3),

$$
\int f_{0}(x)(\psi(x)-\phi(x)) \lambda(d x) \leq 0
$$

for all $\psi \in M_{0}[G]$, where $f_{0}=\sum_{i} \alpha_{i} v_{i}$,

thus $f_{0} \in G^{*}=F(\phi)^{*}$. Further $f_{0} \neq 0$, since $\alpha_{i} \neq 0$ for some $i$. Hence, (4.12) implies that $\lambda(D)=0$, where $D=\left\{x \in X: f_{0}(x)=0\right\}$. Finally, (see also the remark preceding Theorem 4.8), (4.15) implies that for almost all $x \notin D$, we have $\phi(x)=1$ or 0 , depending on whether $f_{0}(x)>0$ or $f_{0}(x)<0$, respectively. Thus $\phi=1_{S}$, where $S=\left\{x \in X: f_{0}(x)>0\right\}$ is an additive set.

4.16. Theorem. Suppose that $m=\operatorname{dim} F<\infty$ and let $\phi \in M_{0}$.

(i) In order that $\phi$ be a uniqueness function (relative to $F$ and $\lambda$ ) it is necessary and sufficient that $\phi$ be $(m-1)$-additive, as defined in 3.10. If $(X, \lambda)$ is nonatomic then the associated set $Z$ as in 3.10 must satisfy $\lambda(Z)=0$, that is, $\phi$ must be an indicator function $\phi=1_{S}$ with $S$ as a special generalized additive set.

(ii) If $\phi$ is a uniqueness function then $\phi$ is s-additive, more precisely, $s$ and the associated $f_{r} \in F(\phi)^{*} \quad(r=0, \ldots, s)$ as in 3.10 can be found so as to satisfy $-1 \leq s \leq m-1$ and

$$
s+1+N(\lambda \mid Z) \leq \operatorname{dim} G \leq m .
$$

Recall from 3.10 that $Z=\left\{x \in X: f_{r}(x)=0\right.$ for $\left.0 \leq r \leq s\right\}$ and further that $G=F(\phi)$ is a Z-determining set of functions. One has $N(\lambda, Z)=0$ if and only if $\lambda(Z)=0$ and then $\phi$ is an indicator function.

4.18. Remark. I have been informed that W. A. Carrington in his unpublished thesis [2] already obtained certain special cases of Theorem 4.16. The main point of Theorem 4.16 is that, relative to an arbitrary measure space $(X, \lambda)$ and a finite set $F$ of functions $f: X \rightarrow \mathbf{R}$, (or at least a set $F$ with $\operatorname{dim} F<\infty$ ), a function $\phi \in M_{0}$ is a uniqueness function if and only if $\phi$ is a generalized additive function. If $\phi$ is a uniqueness function (and thus a generalized additive function) but not an indicator function then the associated set $Z$ satisfies $1 \leq$ $N(\lambda \mid Z)<\infty$. The latter is only possible when $(Z, \lambda)$ is purely atomic, in particular, $(X, \lambda)$ must have atoms. In this way we again find (Lemma 4.4) that all uniqueness functions are indicator functions when $(X, \lambda)$ is nonatomic and $\operatorname{dim} F<\infty$.

4.19. Proof of Theorem 4.16. In view of Definition 3.10, Remark 3.14, and Theorem 3.17, it only remains to prove the necessity part of (i). Thus, let $\phi \in M_{0}$ be a given uniqueness function relative to $\lambda$ and $F$ and put $G=F(\phi)$ 
and $k=\operatorname{dim} G$, thus, $1 \leq k \leq m$. Obviously, $G(\phi)=G$, (where $G(\phi)$ is defined by (2.7), but with $F$ replaced by $G$ ), while $\phi$ is also a uniqueness function relative to $\lambda$ and $G$. We must show that $\phi$ is $s$-additive, such that $s$ and the corresponding set $Z$ (as in 3.10) satisfy (4.17). The proof will be by induction with respect to $k \quad(1 \leq k<\infty)$. Let $k \geq 1$ and suppose the theorem is true for situations where the positive integer $k^{\prime}$ on hand is strictly smaller than $k,($ a void assumption when $k=1)$.

Let $y^{0}=y(\phi)$ be the associated reduced moment point defined by (4.3), depending on the particular choice of the $v_{i} \in G(i=1, \ldots, k)$ as in 4.1. One has $y^{0} \in Y=Y_{G}$ with $Y$ as the convex subset of $\mathbf{R}^{k}$ defined by (4.7). From Theorem 5.8, if $y^{0}$ is not a boundary point of $Y$ then $(X, \lambda)$ is discrete with precisely $m$ atoms, (equivalently, $N(\lambda \mid X)=m$ ), while $G^{*}=F^{*}$ consists of all (measurable) functions $f: X \rightarrow \mathbf{R}$, (that is, $G$ is $X$-determining). Hence, see 3.10, the function $\phi$ is $s$-additive with $s=-1$ and associated set $Z=X$, so that (4.17) is satisfied.

It remains to consider the situation that $y^{0} \in \partial Y$. In that case, just as in the proof of Theorem 4.11, there must exist numbers $\alpha_{i}(i=1, \ldots, k)$ not all zero satisfying (4.15). Letting $f_{0}=\sum_{i} \alpha_{i} v_{i}$, thus $f_{0} \in G^{*}$ and $f_{0} \neq 0$, it follows from (4.15) that

$$
\phi(x)=1 \quad \text { if } f_{0}(x)>0 ; \quad \phi(x)=0 \quad \text { if } f_{0}(x)<0,
$$

for almost $[\lambda]$ all $x \in X$ with $f_{0}(x) \neq 0$. Let

$$
X_{0}=\left\{x \in X: f_{0}(x)=0\right\}, \quad \text { where } f_{0}=\sum_{i} \alpha_{i} v_{i} .
$$

If $\lambda\left(X_{0}\right)=0$ then $\phi=1_{S}$ is 0 -additive while $N\left(\lambda \mid X_{0}\right)=0$, hence, (4.17) holds with $s=0$ and $Z=X_{0}$.

Thus, one may as well assume that $\lambda\left(X_{0}\right)>0$. Let further $\phi_{0}: X_{0} \rightarrow \mathbf{R}$ be the restriction of the given uniqueness function $\phi: X \rightarrow \mathbf{R}$ to the subset $X_{0}$ of $X$. Let further

$$
F_{0}=G_{0}=\left\{f_{X_{0}}: f \in G\right\},
$$

where $f_{X_{0}}: X_{0} \rightarrow \mathbf{R}$ denotes the restriction of $f: X \rightarrow \mathbf{R}$ to $X_{0}$. Then $\int\left|f_{0}\right| \phi_{0} d \lambda_{0}<\infty$ for all $f_{0} \in G_{0}$, where $\lambda_{0}$ denotes the restriction of $\lambda$ to $X_{0}$. Interpreted relative to $\left(X_{0}, \lambda_{0}\right)$, one has $F_{0}\left(\phi_{0}\right)=G_{0}$, (analogous to the above $G(\phi)=G)$.

It is convenient to associate to each $\psi_{0}: X_{0} \rightarrow \mathbf{R}$ the unique extension $\psi: X \rightarrow \mathbf{R}$ of $\psi_{0}$ such that $\psi(x)=\phi(x)$ for all $x \in X / X_{0}$. If $0 \leq \psi_{0} \leq 1$ then $0 \leq \psi \leq 1$. Thus $\phi$ is precisely the extension associated to $\phi_{0}$. Using such extensions and the fact that $\phi$ is a uniqueness function relative to $(X, \lambda, G)$, it easily follows that $\phi_{0}$ is a uniqueness function relative to $\left(X_{0}, \lambda_{0}, G_{0}\right)$. Here $k_{0}:=\operatorname{dim}\left(G_{0} \mid X_{0}\right)<k=\operatorname{dim}(G \mid X)$. After all, from (4.21), the basis functions $v_{i} \in G(i=1, \ldots, k)$ for $G^{*}=\operatorname{span} G$ become linearly dependent when 
restricted to $X_{0}$. If $k_{0}=0$ then $\phi_{0}$ could not possibly be a uniqueness function. Hence, in the present situation one has $k_{0} \geq 1$, thus $k \geq 2$.

From the above induction assumption, the assertions of the Theorem will hold with $X, \lambda, \phi$ and $F, G$, respectively, replaced by $X_{0}, \lambda_{0}, \phi_{0}$ and $F_{0}$, $G_{0}$, respectively, $\left(F_{0}=G_{0}\right)$. Thus, the uniqueness function $\phi_{0}$, relative to $\left(X_{0}, \lambda_{0}, G_{0}\right)$, is $t$-additive with a corresponding subset $Z$ of $X_{0}$ as described in Definition 3.10 (this time instead relative to $X_{0}, \lambda_{0}, \phi_{0}$, and $G_{0}$ ). Here, $t$ and $Z$ satisfy

$$
t+1+N\left(\lambda_{0} \mid Z\right) \leq \operatorname{dim}\left(G_{0} \mid X_{0}\right)=k_{0} \leq k-1 .
$$

More precisely, there exist $g_{0}, \ldots, g_{t} \in G_{0}^{*}$ such that the following is true. Here,

$$
\begin{aligned}
S_{0} & =\left\{x \in X_{0}: g_{q}(x) \text { for } 0 \leq q<r ; g_{r}(x)>0 ; \text { for some } r=0, \ldots, t\right\} ; \\
T_{0} & =\left\{x \in X_{0}: g_{q}(x) \text { for } 0 \leq q<r ; g_{r}(x)<0 ; \text { for some } r=0, \ldots, t\right\} ; \\
Z & =\left\{x \in X_{0}: g_{r}(x)=0 \text { for } r=0, \ldots, t\right\},
\end{aligned}
$$

thus, $X_{0}$ is the disjoint union of $S_{0}, T_{0}$, and $Z$. First $\phi_{0}(x)=1$ if $x \in S_{0}$ and $\phi_{0}(x)=0$ if $x \in T_{0}$, (at least a.e. [ $[\lambda]$ ). Moreover, $G_{0}$ is $Z$-determining (see 3.10). Since $G_{0}$ is the restriction of $G$ to $X_{0}$, see (4.22), and $\lambda_{0}$ is the restriction of $\lambda$ to $X_{0}$, this implies that $G$ is $Z$-determining. Letting

$$
S=\left\{x \in X: f_{0}(x)>0\right\} \cup S_{0} ; \quad T=\left\{x \in X: f_{0}(x)<0\right\} \cup T_{0},
$$

we have from (4.20) and (4.21) that $X$ is the disjoint union of $S, T$, and $Z$. Moreover, $\phi(x)=1$ if $x \in S$, while $\phi(x)=0$ if $x \in T$.

Let $f_{0} \in G^{*}$ be as above. For $r=1, \ldots, t+1$, choose $f_{r}$ as any fixed function $f_{r}: X \rightarrow \mathbf{R}$ such that $f_{r} \in G^{*}=F(\phi)^{*}$ and that further the restriction of $f_{r}$ to $X_{0}$ coincides with $g_{r-1} \in G_{0}^{*}$. This is always possible in view of (4.22). Using (4.23), (4.24), one now easily verifies that the subsets $S, T$, and $Z$ of $X$ have precisely the structure (3.11) relative to $\left\{f_{0}, f_{1}, \ldots, f_{s}\right\}$, where $s=t+1$. This proves that $\phi$ is $s$-additive. Moreover, from (4.23) and $N(\lambda \mid Z)=N\left(\lambda_{0} \mid Z\right)$,

$$
s+1+N(\lambda \mid Z) \leq k_{0}+1 \leq k=\operatorname{dim} G \leq m,
$$

thus, also (4.17) is satisfied.

4.26. Remark. Theorem 4.16 can be improved if $X$ is a finite set. Namely, then any generalized additive function is already 0 -additive. Hence, from Theorem 4.16, if $X$ is finite then $\phi \in M_{0}$ is a uniqueness function if and only if $\phi$ is 0 -additive. The latter result was already obtained in [11] by a more direct proof.

After all, let $\phi \in M_{0}$ be $s$-additive and let $f_{r} \in F(\phi)^{*} \quad(r=0, \ldots, s)$ and $Z$ be as in 3.10, thus, $F(\phi)$ is $Z$-determining. Now consider $g=f_{0}+$ $\rho f_{1}+\cdots+\rho^{s} f_{s}$ with $\rho>0$ so small that $\left|f_{q}(x)\right|<\left(\rho^{-1}-1\right)\left|f_{r}(x)\right|$, for all 
$x \in X$ and $0 \leq r \leq q \leq s$ such that $f_{r}(x) \neq 0$. Then $g \in F(\phi)^{*}$ and $Z=\{x \in X: g(x)=0\}$. Moreover, $g(x)>0$ or $g(x)<0$, respectively, implies $\phi(x)=1$ or $\phi(x)=0$, respectively, showing that indeed $\phi$ is also 0 -additive.

4.27. Optimization. Theorem 4.16 also has important consequences for optimization problems as in 2.22 , in the special case that $F$ is spanned by finitely many functions. Equivalently, $\psi \in M_{0}$ in 2.22 is essentially subject to only finitely many moment conditions. For convenience assume that $(X, \lambda)$ has no atoms. It follows from Theorem 4.16 that an optimal function $\phi$ as in 2.22 is unique if and only if $\phi=1_{S}$ with $S$ as a special generalized additive set relative to $\left(X, \lambda, F_{h}\right)$, where $F_{h}=F \cup\{h\}$. Thus $S$ has the structure described in 3.10 , for some $s \geq 0$, even with $\lambda(Z)=0$, except that there $F$ is to be replaced by $F_{h}$. One example would be

$$
\begin{aligned}
S=\left\{x \in X: g_{0}(x)>0 \text { or } g_{0}(x)=0 ;\right. \\
\left.\qquad g_{1}(x)>0 \text { or } g_{0}(x)=g_{1}(x)=0 ; g_{2}(x)>0\right\} .
\end{aligned}
$$

Here, the $g_{i}=f_{i}+\beta_{i} h \in F_{h}^{*} \quad\left(f_{i} \in F^{*}\right.$ and $\left.\beta_{i} \in \mathbf{R} ; i=0,1,2\right)$ are such that $\lambda(Z)=0$, where $Z=\left\{x \in X: g_{0}(x)=g_{1}(x)=g_{2}(x)=0\right\}$. If $\phi=1_{S}$ happens to be optimal then it is the only optimal function.

\section{INDICATOR FUNCTIONS}

Let $k$ be either a positive integer or else $k=+\infty$. In this section, we study sufficient conditions in order that $(X, \lambda, F)$ be $k$-rich. When $k=1$ we also speak of $(X, \lambda, F)$ being rich.

5.1. Definition. The system $(X, \lambda, F)$ is said to be rich, if for each $\phi \in M_{0}$, there exists an indicator function $1_{T} \in M_{0}(\phi)$. Here, $M_{0}(\phi)$ was defined in (2.9).

Equivalently, $(X, \lambda, F)$ is rich if for each function $0 \leq \phi \leq 1$ there exists a subset $T$ of $X$ having the same moments as $\phi$ (relative to $F$ ), in the sense that $\int_{T} f d \lambda=\int f \phi d \lambda$, for all $f \in F(\phi)$, that is, all $f \in F$ such that $\int|f| \phi d \lambda<\infty$. If there exists only one such set $T$ then $T$ would be a weak set of uniqueness, see 2.11 , (but not conversely, see the remarks preceding Lemma 2.13).

We will say that $(X, \lambda, F)$ is $k$-rich if, for each $\phi \in M_{0}$, which is not of the special form $\phi=1_{S}$, with $S$ as a set of uniqueness, the set $M_{0}(\phi)$ contains at least $k$ distinct indicator functions.

Thus $k$-rich implies rich, (which is the same as 1-rich). Further, if $(X, \lambda, F)$ is 2-rich then every weak set of uniqueness is a set of uniqueness; the converse is false, see 5.2. When $F$ is trivial then $M_{0}(\phi)=M_{0}$ for all $\phi \in M_{0}$, thus, $(X, \lambda, F)$ is $k$-rich with $k \geq 2$ as the number of ( $\lambda$-essentially) distinct subsets of $(X, \lambda)$.

5.2. Remarks. Recall from 2.8 that $\psi \in M_{0}(\phi)$ implies $M_{0}(\phi) \supset M_{0}(\psi)$. Hence, in order that $M_{0}(\phi)$ contains $\geq k$ indicator functions it suffices that 
$M_{0}(\psi)$ contains $\geq k$ indicator functions, for some $\psi \in M_{0}(\phi)$. Consequently, in order that $(X, \lambda, F)$ be $k$-rich, it is necessary and sufficient that, for each nonindicator function $\psi \in M_{0}$ the set $M_{0}(\psi)$ contains $\geq k$ indicator functions. After all, if the convex set $M_{0}(\phi)$ does not contain any nonindicator function $\psi$ (such as the average of two distinct indicator functions) then $M_{0}(\phi)=\left\{1_{S}\right\}$, that is, $\phi=1_{S}$ with $S$ as a set of uniqueness.

An important example of a rich system $(X, \lambda, F)$ is one where $(X, \lambda)$ is nonatomic and, moreover, $F$ is finite, (or else is spanned by a finite set). That this system is rich follows easily from a slight modification of the proof of Lemma 4.4 (which itself uses Lyapunov's theorem). If the $v_{i}$ are not all $\lambda$ integrable then note that the subset $S_{r}$ of $X_{r}$ can be chosen so as to duplicate $\phi_{r}$ as to the integrals relative to the $2 k$ function $v_{i}$ and $\left|v_{i}\right|(i=1, \ldots, k)$. Actually, the system on hand is even $\infty$-rich, see 5.18 .

The term 'rich' was first used in [5] relative to a special case of the projection type, analogous to the one discussed in $\S 6$. If $(X, \lambda)$ is a finite discrete space then $(X, \lambda, F)$ is never rich, unless $F$ is trivial. After all, let $f \in F$ be fixed with $f \neq 0$. On the one hand, $\alpha=\int f \phi d \lambda$ has an uncountable range, when $\phi$ can be any function satisfying $0 \leq \phi \leq 1$ and $\int|f| \phi d \lambda<\infty$. On the other hand, $\beta=\int_{S} f d \lambda$ assumes only finitely many different values when $S$ ranges over the finitely many different subsets of $(X, \lambda)$.

There exist systems $(X, \lambda, F)$, having $(X, \lambda)$ as a finite discrete space and $F$ nontrivial, (hence, the system is not even rich), such that every weak set of uniqueness is also a set of uniqueness. For instance, let $X=\{1, \ldots, m\}$ with $\lambda(\{i\})=1$, for all $i$, and let $F=\{1\}$, thus, $F^{*}$ consist of the constant functions. Then the only weak sets of uniqueness are $S=X$ and the empty set, each of which is also a set of uniqueness. The following result is an obvious consequence of the definitions involved.

5.3. Lemma. If $(X, \lambda, F)$ is rich then every uniqueness function $\phi \in M_{0}$ is necessarily an indicator function.

5.4. The converse of Lemma 5.3 is false. For instance, let $(X, \lambda)$ be a finite discrete space with at least two atoms and let $F=\{1\}$. Then $\phi \equiv 0$ and $\phi \equiv 1$ are the only uniqueness functions but nevertheless $(X, \lambda, F)$ is not rich.

5.5. Definition. We say that the system $(X, \lambda, F)$ is strongly rich if, for each choice of the subset $D$ of $X$ with $\lambda(D)>0$ and the function $\phi \in M_{0}$, there exists a nonzero bounded function $\theta: X \rightarrow \mathbf{R}$ supported by $D$, (that is, $\theta(x)=0$ if $x \notin D)$, which satisfies

$$
\int|f \theta| d \lambda<\infty ; \quad \int f \theta d \lambda=0, \quad \text { for all } f \in F(\phi) .
$$

Equivalently, $(X, \lambda, F)$ is strongly rich if and only if the set $F(\phi)$ is not $D$ determining (see 3.10), for all choices of $D$ and $\phi$ with $\lambda(D)>0$ and $\phi \in M_{0}$. If $F$ itself is trivial (e.g. $F=\{0\})$ then $(X, \lambda, F)$ is strongly rich in a trivial manner. 
5.7. Remark. Since $\lambda$ is $\sigma$-finite, one may as well assume that $0<\lambda(D)<\infty$. In many applications, (5.6) would be implied by the analogous condition, where $F(\phi)$ is replaced by a quite different and often much smaller class of functions. Sometimes this can be seen from the fact that, for $0<\lambda(D)<\infty$ and $\theta$ as above, the collection, of all $f: X \rightarrow \mathbf{R}$ satisfying $\int f \theta d \lambda=0$, is a linear set which is closed under dominated limits $f=\lim _{r} f_{r}$, that is, $h: X \rightarrow \mathbf{R}$ exists such that $\left|f_{r}(x)\right| \leq h(x) \quad(x \in D$ and all $r)$ and $\int_{D}|h| d \lambda<\infty$.

The term 'strongly ri 2 ' was first used in [5], relative to a class $F$ of the type discussed in $\S 6$. Definition 5.5 goes back to Kingman and Robertson [13] who instead speak of $F$ being thin relative to $(X, \lambda)$. But note that in $[5,13]$ it is always assumed that $1 \in F$ and, moreover, that each $f \in F$ is $\lambda$-integrable, in which case $\lambda(X)<\infty$ and $F(\phi)=F$ for all $\phi \in M_{0}$.

The $\lambda$ is strongly rich relative to $F$ then so is any measure $\rho(d x)=$ $q(x) \hat{\lambda}(d x)$, as long as (in an obvious notation) $F_{\lambda}(\phi) \supset F_{\rho}(\phi)$, for all $\phi \in$ $M_{0}$. For instance, the latter condition is satisfied (with $F_{\lambda}(\phi)=F_{\rho}(\phi)=F$ ) when each $f \in F$ is both $\lambda$-integrable and $\rho$-integrable. See [5] for the easy proof; (hint: choose $\theta=h / q$ where $h$ is a bounded function supported by $\{x \in D: q(x) \geq \delta>0\}$ such that $\int f h d \lambda=0$ for all $\left.f \in F_{\lambda}(\phi)\right)$.

5.8. Remark. Let $W$ be the maximal set, such that $f(x)=0$ a.e. on $W$, for each $f \in F$. If $\lambda(W)=0$ we will say that $(X, \lambda, F)$ is regular or also that it is nonsingular. It would be sufficient for regularity that $1 \in F$.

If $F$ is trivial then $W=X$ thus $(X, \lambda, F)$ is singular. Every time that $(X, \lambda, F)$ is singular, that is, $\lambda(W)>0$, then there cannot exist any uniqueness function at all. After all, changing the values $\phi(x) \quad(x \in W)$ has no influence whatsoever on the integrals $\int f \phi d \lambda(f \in F)$. Since $W$ plays no real role, it would be natural to restate the uniqueness problem on hand by simply dropping $W$ from $X$, thereby achieving regularity.

The restriction of $(\lambda, F)$ to $W$ is strongly rich in a trivial way, (whether or not $W$ possesses atoms). On the other hand, if $(X, \lambda)$ has any atom $x_{0}$ in $W^{c}=X / W$ then $(X, \lambda, F)$ fails to be strongly rich. After all, apply Definition 5.5 with $D=\left\{x_{0}\right\}$ and $\phi=0$, observing that $x_{0} \notin W$ implies $f\left(x_{0}\right) \neq 0$, for some $f \in F=F(0)$. In particular, if $(X, \lambda, F)$ is both strongly rich and regular then $(X, \lambda)$ must be nonatomic.

Conversely, if $(X, \lambda)$ is nonatomic then $(X, F, \lambda)$ is strongly rich provided $F=\left\{h_{1}, \ldots, h_{m}\right\}$ is finite; (naturally, all that matters is the condition $\left.\operatorname{dim} F^{*}<\infty\right)$. This result is due to Kingman and Robertson [13, Lemma 2]. As a somewhat different proof, if $X$ is nonatomic and $\lambda(D)>0$, then there certainly exist $m+1$ disjoint subsets $D_{0}, \ldots, D_{m}$ of $D$ each of positive $\lambda$ measure, such that each $h_{j}$ is bounded on each $D_{k}$. Let $a_{j k}=\int_{D_{k}} h_{j} d \lambda$ and choose $z_{k}(k=0,1, \ldots, m)$ as constants not all zero such that $\sum_{k} a_{j k} z_{k}=0$ for $j=1, \ldots, m$. Then $\theta=\sum_{k} z_{k} 1_{D_{k}}$ is a nonzero bounded function which is supported by $D$ and satisfies (5.6). 
Since $(X, \lambda,\{0\})$ is trivially strongly rich, the following is a more general result. Namely, if $(X, \lambda)$ is nonatomic and $(X, \lambda, F)$ is strongly rich then also $(X, \lambda, G)$ is strongly rich, provided $G$ can be obtained from $F$ by adjoining finitely many functions $h_{j}: X \rightarrow \mathbf{R},(j=1, \ldots, m)$. That is, $G=F \cup$ $\left\{h_{1}, \ldots, h_{m}\right\}$ in which case $G^{*}$ consists of all functions of the form $f+\sum_{j} \beta_{j} h_{j}$ with $f \in F^{*}$ and $\beta_{j} \in \mathbf{R}$.

As to the proof of the latter assertion, let $\phi \in M_{0}$ be given and let $D, D_{k}$ be as above. For $k=0, \ldots, m$, choose $\theta_{k}$ as a bounded nonzero function supported by $D_{k}$ which satisfies (5.6). Let $a_{j k}=\int r_{j} \theta_{k} d \lambda$ and choose the $z_{k}$ as above. Then $\theta=\sum_{k} z_{k} \theta_{k}$ is a bounded nonzero function supported by $D$ which satisfies (5.6) with $F(\phi)$ replaced $F(\phi) \cup\left\{h_{1}, \ldots, h_{m}\right\}$, hence, also with $F(\phi)$ replaced by $G(\phi)$. Observe that in the present proof we did not use Lyapunov's theorem, (as we did in 4.5 and 5.2).

Propositions 5.9 and 5.13 below are generalizations of known results due to Kingman and Robertson [13] and Gutmann, Kemperman, Reeds, and Shepp [5].

5.9. Proposition. Suppose that $(X, \lambda, F)$ is strongly rich. We assert that:

(i) Each uniqueness function is necessarily an indicator function.

(ii) Let $\phi \in M_{0}$ and $\psi \in M_{0}(\phi)$. Let $E_{0}(\phi)$ denote the (possibly empty) set of extreme points of the convex set $M_{0}(\phi)$. Then in order that $\psi$ be an indicator function it is necessary and sufficient that $\psi \in E_{0}(\phi)$.

(iii) Suppose $(X, \lambda)$ is nonatomic and let $\phi \in M_{0}$. Then either $\phi$ is a uniqueness function or else $M_{0}(\phi)$ is infinite dimensional. That is, $\operatorname{dim} M_{0}(\phi)=$ 0 or $+\infty$.

5.10. Proof. If $\phi$ is a uniqueness function then $M_{0}(\phi)=\{\phi\}$, hence, $\phi$ is an extreme point of $M_{0}(\phi)$. Therefore, (ii) implies (i). As to (ii), note first that any indicator function $1_{S}$ is trivially an extreme point of $M_{0}$ and, hence, of any (convex) set $M_{0}(\phi)$ which contains $1_{S}$. As to the converse, let $\phi \in M_{0}$ and $\psi \in M_{0}(\phi)$ and suppose that $\psi$ is not an indicator function. Then there exists $\varepsilon>0$ such that $\lambda(D)>0$, where $D=\{x \in X: \varepsilon \leq \psi(x) \leq 1-\varepsilon\}$. Let $\theta$ be as in 5.5. One may assume that $|\theta| \leq \varepsilon$. Clearly, $\psi \pm \theta \in M_{0}(\phi)$, showing that $\psi$ cannot be extreme in $M_{0}(\phi)$. This proves (ii).

As to (iii), since $(X, \lambda)$ is nonatomic, the above set $D$ contains an infinite collection of disjoint subsets $D_{1}, D_{2}, \ldots$, each of positive $\lambda$-measure. Each $D_{i}$ has a corresponding nonzero function $\theta_{i}$ as above (supported by $D_{i}$ ). The resulting functions $\psi+\theta_{i} \in M_{0}(\phi) \quad(i=1,2, \ldots)$ are easily seen to be linearly independent. Consequently, $M_{0}(\phi)$ is infinite dimensional as soon as it contains at last one nonindicator function $\psi$, (such as the average of two distinct indicator functions). The only alternative is that $M_{0}(\phi)$ consists of a single indicator function $1_{S}$, that is, $\phi=1_{S}$ is a uniqueness function. By the way, since the system $(X, \lambda, F)$ was already assumed to be strongly $\operatorname{rich},(X, \lambda)$ will be nonatomic as soon as $(X, \lambda, F)$ is regular, (see 5.8). 
5.11. Corollary. Suppose that $(X, \lambda, F)$ is strongly rich and let $1 \leq k \leq \infty$. Then $(X, \lambda, F)$ is $k$-rich if and only if $M_{0}(\phi)$ possesses at least $k$ extreme points, for each $\phi \in M_{0}$ which is not a uniqueness function. On the other hand, if $\phi$ is a uniqueness function, (that is, $M_{0}(\phi)=\{\phi\}$ ), then $\phi=1_{S}$ for some subset $S$ of $X$.

5.12. Proof. This follows immediately from Definition 5.1 and Proposition 5.9 .

5.13. Proposition. Suppose that each $f \in F$ is $\lambda$-integrable. Let $\phi \in M_{0}$ be fixed thus $F(\phi)=F$.

Assertion. The subset $M_{0}(\phi)$ of the dual $L^{\infty}$ of $L^{1}$ is the weak ${ }^{*}$-closed convex hull of the set $E_{0}(\phi)$ of its extreme points. Hence, $M_{0}(\phi)$ possesses at least $1+\operatorname{dim} M_{0}(\phi)$ extreme points. Consequently, either $M_{0}(\phi)$ possesses at least two extreme points or else $\phi$ is a uniqueness function.

5.14. Proof. Here $L^{\infty}=L^{\infty}(X, \lambda)$ and $L^{1}=L^{1}(X, \lambda)$. It is assumed that $L^{1} \supset F$. Let us assign to $L^{\infty}$ the weak* topology, that is, the coarsest topology such that $\phi \rightarrow \int f \phi d \lambda$ is continuous on $L^{\infty}$ for each $f \in L^{1}$. Let $\phi \in M_{0}$. From Alaoglu's theorem the set $M_{0}=\left\{\psi \in L^{\infty}: 0 \leq \psi \leq 1\right\}$ is weak*-compact, hence, so is the nonempty weak*-closed and convex subset

$$
M_{0}(\phi)=\left\{\psi \in M_{0}: \int f \psi d \lambda=\int f \phi d \lambda \text { for all } f \in F\right\} .
$$

It follows from the Krein-Milman theorem that $M_{0}(\phi)$ is the weak*-closed convex hull of the set $E_{0}(\phi)$ of all extreme points of $M_{0}(\phi)$.

5.15. Theorem. Suppose that each $f \in F$ is $\lambda$-integrable and further that $(X, \lambda, F)$ is strongly rich. We assert that:

(i) Each $(X, \lambda, F)$ uniqueness function is also an indicator function.

(ii) The system $(X, \lambda, F)$ is 2-rich and thus rich.

(iii) The system $(X, \lambda, F)$ is $\infty$-rich as soon as it is nonatomic or regular.

5.16. Proof. Here, (i) follows from Proposition 5.9. Consider $\phi \in M_{0}$ which is not a uniqueness function. By Proposition 5.9, the set $E_{0}(\phi)$ of extreme points of $M_{0}(\phi)$ coincides with the set of indicator functions $1_{T} \in M_{0}(\phi)$. Therefore, Proposition 5.13 implies that $(X, \lambda, F)$ is 2-rich. And even $\infty$ rich provided $\operatorname{dim} M_{0}(\phi)=\infty$. By Proposition 5.9, the latter is true as soon as $(X, \lambda)$ is nonatomic. Also recall (see 5.8) that a strongly rich system is nonatomic as soon as it is regular.

5.17. Remark. The result that under the assumptions stated, $(X, \lambda, F)$ must be rich, is due to Kingman and Robertson [13, p. 349]. They also noted that $(X, \lambda, F)$ being strongly rich is also a necessary condition in the following sense. Assume that each $f \in F$ is $\lambda$-integrable, thus, $F(\phi)=F$ for all $\phi \in M_{0}$. For each subset $D$ of $X$ with $\lambda(D)>0$, let $\left(D, \lambda_{D}, F_{D}\right)$ denote the restriction of $(X, \lambda, F)$ to $D$. Then the system $(X, \lambda, F)$ is strongly rich if and only if each system $\left(D, \lambda_{D}, F_{D}\right)$ is rich. 
For, on the one hand, if $(X, \lambda, F)$ is strongly rich then also $\left(D, \lambda_{D}, F_{D}\right)$ is strongly rich and thus rich, in view of Theorem 5.15. Conversely, suppose $(X, \lambda, F)$ is not strongly rich. Then by Definition 5.5 , there exists a subset $D$ of $X$ with $\lambda(D)>0$ such that $F$ is $D$-determining. But then each function $0 \leq \phi \leq 1$ on $D$ is a uniqueness function relative to $\left(D, \lambda_{D}, F_{D}\right)$, showing that the latter system is not rich.

Theorem 5.18 below may be regarded as a strengthening of certain versions of Lyapunov's theorem, such as the one given by Karlin [7, p. 266]. Lyapunov's theorem is never used in the proof, see also 5.8, though the proof could be shortened by using it. See Kingman and Robertson [13] for a generalization of Lyapunov's theorem in a different direction.

5.18. Theorem. Suppose that $(X, \lambda)$ is nonatomic and further that $F$ is finite (or else that $\left.\operatorname{dim} F^{*}<\infty\right)$. Then $(X, \lambda, F)$ is $\infty$-rich.

5.19. Proof. Let $F=\left\{f_{1}, \ldots, f_{m}\right\}$. We already observed in 5.8 that $(X, \lambda, F)$ is strongly rich. Suppose first that each $f_{i}$ is $\lambda$-integrable. Then it follows from Theorem 5.15 that $(X, \lambda, F)$ is $\infty$-rich. In other words, if $\phi$ is a nonindicator function and $f_{1}, \ldots, f_{m}$ are finitely many $\lambda$-integrable functions then there exist infinitely many different subsets $S$ of $X$ such that

$$
\int_{S}\left|f_{i}\right| d \lambda<\infty ; \quad \int_{S} f_{i} d \lambda=\int_{X} f_{i} \phi d \lambda \quad(i=1, \ldots, m) .
$$

One can even attain that, in addition, $\int_{S}\left|f_{i}\right| d \lambda=\int_{X}\left|f_{i}\right| \phi d \lambda \quad(i=1, \ldots, m)$.

Now, let $F=\left\{f_{1}, \ldots, f_{m}\right\}$ be an arbitrary finite set of functions and let $\phi \in M_{0}$ be a fixed nonindicator function. Let $G=F(\phi)=\left\{g_{1}, \ldots, g_{k}\right\}$, where $k \leq m<\infty$, thus, $\int\left|g_{i}\right| \phi d \lambda<\infty \quad(i=1, \ldots, k)$. Decompose $X$ into at most countably many disjoint sets $X_{r}$, with $0<\lambda\left(X_{r}\right)<\infty$ and such that each $g_{i}$ is bounded on each $X_{r}$. Let $\phi_{r}$ be the restriction of $\phi$ to $X_{r}$. For some indices, $r, \phi_{r}$ might be the indicator function of a subset $S_{r}$ of $X_{r}$. Next, let $r$ be such that $\phi_{r}$ is not an indicator function, (there must be at least one such index $r$ ). From the previous result, with $X$ and $f_{i}$ replaced by $X_{r}$ and $g_{i}$, there must exist infinitely many different subsets $S_{r}=S_{r, t}(t=1,2, \ldots)$ of $X_{r}$ such that

$$
\int_{S_{r}}\left|g_{i}\right| d \lambda=\int_{X_{r}}\left|g_{i}\right| \phi d \lambda ; \quad \int_{S_{r}} g_{i} d \lambda=\int_{X_{r}} g_{i} \phi d \lambda, \quad \text { for all } i=1, \ldots, k .
$$

Let $S$ be the union of the $S_{r}$. Each such union $S$ satisfies

$$
\int_{S}\left|g_{i}\right| d \lambda=\int_{X}\left|g_{i}\right| \phi d \lambda<\infty ; \quad \int_{S} g_{i} d \lambda=\int_{X} g_{i} \phi d \lambda \quad(i=1, \ldots, k),
$$

thus $1_{S} \in M_{0}(\phi)$. Since the above construction leads to infinitely many possible sets $S$, this implies that $M_{0}(\phi)$ contains infinitely many different indicator functions, showing that $(X, \lambda, F)$ is $\infty$-rich.

5.20. Theorem. Let $\phi \in M_{0}$ be fixed and suppose that the system $(X, \lambda, F(\phi))$ is strongly rich. Then if $\phi$ is a uniqueness function it must be an indicator function. 
Next, assume that $X$ is a countable union of sets $X_{r}(\phi)$, each of finite $\lambda$ measure, such that the restriction of $f$ to $X_{r}$ is $\lambda$-integrable, for each $f \in F(\phi)$ and each $r$. Also suppose that $(X, \lambda)$ is nonatomic and that even $\left(X, \lambda, G_{\phi}\right)$ is strongly rich. Here, $G_{\phi}$ denotes the set of all functions $f$ and $|f|$ with $f \in F(\phi)$. Assertion. Unless $\phi=1_{S}$ with $S$ as a set of uniqueness, there exist finitely many different subsets $S$ of $X$ such that $1_{S} \in M_{0}(\phi)$.

5.21. Proof. Since $\phi \in M_{0}$ is fixed, one may as well assume that $F=F(\phi)$. The proof of Theorem 5.10 is completely analogous to the proof in 5.19 of Theorem 5.18. Simply apply Theorem 5.15 to the restriction of $(X, \lambda, F(\phi))$ to the different subsets $X_{r}(\phi)$ of $X$.

5.22. Remark. Theorem 5.20 generalizes Theorem 5.15. After all, see 5.8, if $(X, \lambda)$ is nonatomic and $\operatorname{dim} G_{\phi}<\infty$ then $\left(X, \lambda, G_{\phi}\right)$ is always strongly rich.

5.23. Optimization. Consider the general optimization problem 2.22 except that now we assume that each $f \in F$ is $\lambda$-integrable as well as the given function $h: X \rightarrow \mathbf{R}$. From 5.14, the set $M_{0}(\phi)$ is weak*-compact while $\psi \rightarrow \int h \psi d \lambda$ defines a weak*-continuous function on $M_{0}(\phi)$. Consequently, optimal functions $\phi$ always exist.

Suppose $(X, \lambda, F)$ is regular (as happens when $1 \in F)$ and strongly rich, thus $(X, \lambda)$ is nonatomic. It follows from a result in 5.8 that also $\left(X, \lambda, F_{h}\right)$ is strongly rich with $F_{h}=F \cup\{h\}$. We conclude from Theorem 5.15 that $\left(X, \lambda, F_{h}\right)$ is $\infty$-rich and thus rich. In particular, an optimal function $\phi$ which is unique is always an indicator function. Moreover, either there is only one optimal function $\phi$ (necessarily an indicator function) or else there exist infinitely many distinct optimal function, each of which is an indicator function. See 6.31 for an important case where $(X, \lambda, F)$ is strongly rich. Another would be the case that $(X, \lambda)$ is nonatomic and $\operatorname{dim} F^{*}<\infty$, see 5.8.

\section{RECONSTRUCTING A MEASURE FROM ITS PROJECTIONS}

Here, we discuss applications of the previous results to measures with given marginals, as weil as related results in the literature. In the sequel $(X, \lambda)$ is a fixed $\sigma$-finite measure space and $\pi=\left\{\pi_{j} ; j \in J\right\}$ a given nonempty set of (measurable) maps $\pi_{j}: X \rightarrow Y_{j}(j \in J)$. Here, the $Y_{j}$ are given measurable spaces. Let $\mu$ be a measure on $X$ such that $\mu \leq \lambda$. We will derive necessary or sufficient conditions in order that a given measure $\mu$ be uniquely determined by its associated set of 'marginals' $\left\{\pi_{j} \mu ; j \in J\right\}$. We are also interested in the collection of sets $S$ such that $1_{S} d \lambda$ has the same marginals as $\mu$.

Let $\mu \leq \lambda$, thus, $d \mu=\phi d \lambda$ with $\phi \in M_{0}$. Its $\pi_{j}$-image $\nu_{j}=\pi_{j} \mu$ is the measure on $Y_{j}$ defined by $\nu_{j}(B)=\mu\left(\pi_{j}^{-1} B\right)$. It will also be referred to as the $\pi_{j}$-marginal of $\phi$ or $\mu$. Knowing this marginal $\nu_{j}$ is the same as knowing the value

$$
\int g_{j}\left(\pi_{j} x\right) \phi(x) \lambda(d x)=\int g_{j}\left(\pi_{j} x\right) \mu(d x)=\int g_{j}(y) \nu_{j}(d y),
$$


for each nonnegative function $g_{j}: Y_{j} \rightarrow \mathbf{R}$. At least when $\nu_{j}$ is $\sigma$-finite, this is the same as knowing the value (6.1) for each $\nu_{j}$-integrable functions $g_{j}: Y_{j} \rightarrow$ $\mathbf{R}$, that is, $g_{j} \in L^{1}\left(Y_{j}, \nu_{j}\right)$. Which is the same as $g_{j}\left(\pi_{j} x\right)$ being integrable relative to $d \mu=\phi d \lambda$.

6.2. Remark. Given a particular marginal $\nu_{j}=\pi_{j} \mu$ of $\mu$, we will only use the resulting information about $\mu$ as expressed by the values (6.1), with $g_{j}: Y_{j} \rightarrow \mathbf{R}$ running through the $\nu_{j}$-integrable functions. Even in very natural settings, it can easily happen that the marginal $\nu_{j}=\pi_{j} \mu$ of $\mu$ is not $\sigma$-finite, in spite of the fact that $\mu$ itself is $\sigma$-finite, (since $\mu \leq \lambda$ where $\lambda$ is $\sigma$-finite). Consider for instance the special case that $X=\mathbf{R}_{+}^{n}$ with $\lambda=m_{n}$ as $n$-dimensional Lebesgue measure. Let further $\pi=\left\{\pi_{1}, \ldots, \pi_{n}\right\}$, where $\pi_{j}: X \rightarrow \mathbf{R}$ is defined by $\pi_{j} x=x_{j}$, with $x_{j}$ as the $j$ th coordinate of $x \in R^{n}$. Here, the $j$ th marginal $\lambda_{j}=\pi_{j} \lambda$ of $\lambda$ satisfies $\lambda_{j}(B)=0$ or $+\infty$, respectively, depending on whether $m_{1}(B)=0$ or $m_{1}(B)>0$, respectively.

Similarly, for many other measures $\mu \leq \lambda$. For instance, let $\mu={ }_{S} d \lambda$ where $S=\left\{x \in X: x_{j} \leq 1\right.$ for $\left.j \geq 2\right\}$ and let $\nu_{j}=\pi_{j} \mu,(j=1, \ldots, n)$. Then $\nu_{1}=m_{1}$ is $\sigma$-finite. On the other hand, for $j \geq 2$, the marginal $\nu_{j}$ of $\mu$ is not $\sigma$-finite, since $\nu_{j}(B)=0$ or $\infty$, depending on whether $m_{1}(B \cap D)=0$ or $>0$, respectively, where $D=[0,1]$. There clearly exist many other measures $\phi d \lambda\left(\phi \in M_{0}\right)$ on $X$ having exactly these same marginals.

6.3. The class $F_{\pi}$. In the sequel, $F=F_{\pi}$ will denote the set of all functions $f: X \rightarrow \mathbf{R}$ defined by

$$
F_{\pi}=\left\{f(x)=g_{j}\left(\pi_{j} x\right) \text { for some } j \in J \text { and some } g_{j}: Y_{j} \rightarrow \mathbf{R}\right\} .
$$

Naturally, $g_{j}$ must also be measurable. Let $\phi \in M_{0}$ and let $d \mu_{\phi}=\phi d \lambda$ be the associated measure on $X$. It follows from (2.7), (6.1), and (6.4) that (6.5)

$$
F_{\pi}(\phi)=\left\{\text { all } f: X \rightarrow \mathbf{R} \text { with } f=g_{j} \pi_{j} \text { for some } j \in J \text { and } g_{j} \in L^{1}\left(\pi_{j} \mu_{\phi}\right)\right\} \text {. }
$$

Note that $F_{\pi}(\phi)$ contains a function $f^{+}>0$ as soon as at least one of the marginals $\pi_{j} \mu_{\phi}(j \in J)$ is $\sigma$-finite.

For each $\phi \in M_{0}$, let $M_{0}(\phi)$ denote the class of functions defined by (2.9) relative to the system $\left(X, \lambda, F_{\pi}\right)$. That is,

$$
M_{0}(\phi)=\left\{\psi \in M_{0}: F_{\pi}(\psi) \supset F_{\pi}(\phi) ; \int f d \mu_{\psi}=\int f d \mu_{\phi} \text {, for all } f \in F_{\pi}(\phi)\right\} .
$$

Equivalently, using (6.1) and (6.5), $M_{0}(\phi)$ consists of all $\psi \in M_{0}$ such that, for all $j \in J$,

if $\int\left|g_{j}\right| d\left(\pi_{j} \mu_{\phi}\right)<\infty$ then $\int\left|g_{j}\right| d\left(\pi_{j} \mu_{\psi}\right)<\infty ; \int g_{j} d\left(\pi_{j} \mu_{\psi}\right)=\int g_{j} d\left(\pi_{j} \mu_{\phi}\right)$.

Finally, let $M_{00}(\phi)$ denote the set of all $\psi \in M_{0}$ such that $\pi_{j} \mu_{\phi}=\pi_{j} \mu_{\psi}$, for all $j \in J$. 
6.8. Remark. For each fixed $j \in J$, condition (6.7) is clearly necessary in order that $\pi_{j} \mu_{\phi}=\pi_{j} \mu_{\psi}$. It is also sufficient provided $\pi_{j} \mu_{\phi}$ is $\sigma$-finite. It follows that $M_{0}(\phi) \supset M_{00}(\phi)$ and further that $M_{00}(\phi)=M_{0}(\phi)$ provided each $\pi_{j} \mu_{\phi}$ is $\sigma$-finite.

For $j \in J$ fixed, condition (6.7) is not always sufficient for $\pi_{j} \mu_{\phi}=\pi_{j} \mu_{\psi}$. This can be seen in a situation, where $\pi_{j} \mu_{\phi}(B)=0$ or $\infty$, depending on whether $B \cap D$ is empty or nonempty, respectively, with $D$ as a fixed nonempty subset of $Y_{j}$. In this case, (6.7) merely requires that $\pi_{j} \mu_{\psi}$ be supported by $D$.

6.9. Uniqueness. As usual, the function $\phi \in M_{0}$ is said to be a uniqueness function relative to the system $\left(X, \lambda, F_{\pi}\right)$ if $M_{0}(\phi)=\{\phi\}$. We will say that $\phi$ is a $\pi$-uniqueness function if $M_{00}(\phi)=\{\phi\}$. Equivalently, $\phi$ is a $\pi$-uniqueness function if there exists no other $\psi \in M_{0}$ such that $d \mu_{\psi}=\psi d \lambda$ has the same $\pi_{j}$-marginal as $d \mu_{\phi}=\phi d \lambda$, for all $j \in J$. A subset $S$ of $X$ is said to be a set of $\pi$-uniqueness when $\phi=1_{S}$ is a $\pi$-uniqueness function.

From $M_{0}(\phi) \supset M_{00}(\phi)$, in order that $\phi \in M_{0}$ be a $\pi$-uniqueness function, it is sufficient that $\phi$ be a uniqueness function relative to $\left(X, \lambda, F_{\pi}\right)$. From Theorem 3.17, a sufficient condition for the latter is in turn that $\phi$ be a generalized additive function relative to $\left(X, \lambda, F_{\pi}\right)$, as defined in 3.10 .

6.10. Generalized additive sets. Let us discuss here only the case $\phi=1_{S}$ with $S$ as a special generalized additive set relative to $\left(X, \lambda, F_{\pi}\right)$. As usual, $F_{\pi}(S)^{*}$ denotes the linear span of $F_{\pi}(S)=F_{\pi}\left(1_{S}\right)$. By 3.10 the subset $S$ of $X$ is a special generalized additive set, (and thus a set of uniqueness relative to $\left(X, \lambda, F_{\pi}\right)$ and thus a set of $\pi$-uniqueness), if one can find finitely many $f_{r} \in F_{\pi}(S)^{*}$ $(r=0, \ldots, s)$, such that $S$ is of the form

$$
S=\bigcup_{r=0}^{s}\left\{x \in X: f_{q}(x)=0 \text { for } 0 \leq q<r ; f_{r}(x)>0\right\},
$$

(up to a set of $\lambda$-measure zero), and such that $\lambda(Z)=0$ where

$$
Z=\left\{x \in X: f_{r}(x)=0 \text { for all } 0 \leq r \leq s\right\} .
$$

In this case, one also says that $S$ is a special $s$-additive set. The subset $S$ is said to be additive if either $S=\{f(x)>0\}$ or $S=\{f(x) \geq 0\}$, for some choice of $f \in F_{\pi}(S)^{*}$, and moreover $f^{+} \in F_{\pi}(S)^{*}$ exists such that $f^{+}(x)>0$ a.e. [ $\lambda$ ] on $Z=\{x \in X: f(x)=0\}$. Letting $f_{0}=f$ and $f_{1}= \pm f^{+}$, we see that any additive set is also a special 1 -additive set.

Observe, from (6.1) and (6.5), that $F_{\pi}(S)^{*}$ consists of all functions $f: X \rightarrow$ $\mathbf{R}$ of the special form

$$
f(x)=\sum_{j \in J} g_{j}\left(\pi_{j} x\right) \text { where } \int_{S}\left|g_{j}\left(\pi_{j} x\right)\right| \lambda(d x)<\infty,
$$

and such that all but finitely many functions $g_{j}: Y_{j} \rightarrow \mathbf{R}$ are identically zero. The last condition (6.13) is equivalent to the condition that $g_{j}$ be integrable relative to the $\pi_{j}$-marginal of $d \mu_{S}=1{ }_{S} d \lambda$. in particular, different sets $S$ with 
the same collection of marginals $\left\{\pi_{j} \mu_{S} ; j \in J\right\}$ also have the same associated set $F_{\pi}(S)$.

6.14. Remark. In trying to construct such special generalized additive sets $S$, one typically first selects functions $f_{r}(x)=\sum_{j \in J} g_{r j}\left(\pi_{j} x\right) \in F_{\pi}^{*}=\operatorname{span}\left(F_{\pi}\right)$ $(r=0, \ldots, s)$, such that $Z$ defined by $(6.11)$ satisfies $\lambda(Z)=0$. Afterwards, define $S$ as in (6.11). Then $S$ is a special generalized additive set (and thus a set of $\pi$-uniqueness), provided $f_{r} \in F_{\pi}(S)^{*}(r=0, \ldots, s)$.

The above definition of a generalized additive set is analogous to our definition in [11], except that there it is always assumed that $\lambda(S)<\infty$. The latter assumption considerably simplifies things. For it implies that $f^{+} \equiv 1$ is an everywhere positive function with $f^{+} \in F_{\pi}(S)$ and further that the measure $d \mu_{S}=1_{S} d \lambda$ is finite and, hence, so are all its marginals $\nu_{j}=\pi_{j} \mu_{S}$. The latter in turn implies that $M_{0}(\phi)=M_{00}(\phi)$, for all $\phi \in M_{0}$, in particular, $\pi$-uniqueness is the same as uniqueness relative to the system $\left(X, \lambda, F_{\pi}\right)$.

6.15. Using the results of $\S 5$, let us now study the question whether there exist one or more sets $S$ such that $d \mu_{S}=1_{S} d \lambda$ has exactly the same marginals as a given measure $\phi d \lambda\left(\phi \in M_{0}\right)$. In the sequel, it will be useful to employ the following condition.

6.16. Condition $\mathrm{L}$. We will say that $(X, \lambda, \pi)$ satisfies Condition $\mathrm{L}$ when, for each $j \in J$, the marginal $\lambda_{j}=\pi_{j} \lambda$ of $\lambda$ (itself a measure on $Y_{j}$ ) is $\sigma$-finite.

6.17. Remarks. It would be sufficient that $\lambda$ itself be finite. Condition $\mathrm{L}$ is an essential restriction, see the example in 6.2. Suppose Condition $\mathrm{L}$ holds. Then we have, for all $\phi \in M_{0}$ and $j \in J$, that also $\pi_{j} \mu_{\phi} \leq \lambda_{j}$ is $\sigma$-finite, consequently (see 6.8) $M_{00}(\phi)=M_{0}(\phi)$. Moreover (Radon-Nikodym), $\nu_{j}=$ $\pi_{j} \mu \leq \lambda_{j}$ has the form $d \nu_{j}=h_{j} d \lambda_{j}$ where $h_{j}: Y_{j} \rightarrow \mathbf{R}$ is such that $0 \leq h_{j} \leq 1$.

6.18. The class $G_{\pi}$. This class will only be used in the presence of Condition L. Thus, assume that all marginals $\lambda_{j}(j \in J)$ of the reference measure $\lambda$ are $\sigma$-finite. For each $j \in J$, let $G_{j}$ be a fixed set of $\lambda_{j}$-integrable functions $g_{j}: Y_{j} \rightarrow \mathbf{R}$, such that $G_{j}$ is a determining class for $\left(Y_{j}, \lambda_{j}\right)$. By this we mean that

(6.19) if $h_{j}: Y_{j} \rightarrow \mathbf{R}$ is bounded; $\int g_{j} h_{j} d \lambda_{j}=0$ for all $g_{j} \in G_{j}$ then $h_{j}=0$,

(a.e. $\left.\left[\lambda_{j}\right]\right)$. Here, $h_{j}$ is fixed. Since $\lambda_{j}$ is a $\sigma$-finite, such a determining class $C_{j}$ always exists. From the last remark 6.17, when $\mu$ is an unknown measure on $X$ known to satisfy $\mu \leq \lambda$, then for each fixed $j \in J$ the projection $\nu_{j}=\pi_{j} \mu$ of $\mu$ onto $Y_{j}$ is uniquely determined by the values of the integral (6.1) with $g_{j}$ ranging through $G_{j}$.

Further, $G=G_{\pi}$ will denote the set of all functions $f: X \rightarrow \mathbf{R}$ of the special form

$$
G_{\pi}=\left\{f(x)=g_{j}\left(\pi_{j} x\right) \text { for some } j \in J \text { and some } g_{j} \in G_{j}\right\} .
$$


Observe that each $f \in G_{\pi}$ is $\lambda$-integrable. Hence, we have for all $\phi \in M_{0}$ that $G_{\pi}(\phi)=G_{\pi}$, (see 2.7). Note that, for many $\phi \in M_{0}$, there will exist functions $g_{j}: Y_{j} \rightarrow \mathbf{R}$ that are integrable relative to $\pi_{j} \mu_{\phi}$ but not relative to $\lambda_{j}$. The corresponding function $f(x)=g_{j}\left(\pi_{j} x\right)$ would belong to $F_{\pi}(\phi)$ but not to $G_{\pi}(\phi)=G_{\pi}$, even when $G_{j}=L^{1}\left(Y_{j}, \lambda_{j}\right)$.

It is important to note (using (6.19)), that for each $\phi \in M_{0}$ the above class $M_{00}(\phi)=M_{0}(\phi)$ coincides with the class $M_{0}(\phi)$ as defined by (2.9), but now relative to the system $\left(X, \lambda, G_{\pi}\right)$ instead of the system $\left(X, \lambda, F_{\pi}\right)$.

We further assert that the present system $\left(X, \lambda, G_{\pi}\right)$ is regular (as defined in 5.8). One must show that $\lambda(W)=0$ when $W$ is a subset of $X$, such that, for all $j \in J$ and $g_{j} \in G_{j}$, one has $g_{j}\left(\pi_{j} x\right)=0$ a.e. [ $\lambda$ ] on $W$. Even a single $j \in J$ would suffice. Let $d \mu=1_{W} d \lambda$. Namely, using (6.1) and the fact that $G_{j}$ is $\left(Y_{j}, \lambda_{j}\right)$-determining, the latter property implies that $\pi_{j} \mu=0$, therefore, $\lambda(W)=\mu(X)=\left(\pi_{j} \mu\right)\left(Y_{j}\right)=0$.

6.21. Remark. Still assuming Condition $\mathrm{L}$, the precise choice of the determining set $G_{j}$ for $\left(Y_{j}, \lambda_{j}\right)$ in (6.20) (one for each $j \in J$ ) is irrelevant. Note that condition (6.19) requires precisely that $G_{j}^{*}=\operatorname{span} G_{j}$ be weakly dense in $L^{1}\left(Y_{j}, \lambda_{j}\right)$, a rather weak condition. One example would be the set $G_{j}=L^{1}\left(Y_{j}, \lambda_{j}\right)$ of all $\lambda_{j}$-integrable $g_{j}: Y_{j} \rightarrow \mathbf{R}$. In this case, $G_{\pi}$ contains, for each $j \in J$, a function $f^{+}>0$ of the form $f^{+}(x)=g_{j}\left(\pi_{j} x\right)$. If $\lambda$ is finite one could also choose $G_{j}=L^{\infty}\left(Y_{j}, \lambda_{j}\right)$. Another example is the class $G_{j}$ of all (bounded) indicator functions $1_{B_{j}}$ of subsets $B_{j}$ of $Y_{j}$ such that $\lambda_{j}\left(B_{j}\right)<\infty$. In the latter example, one could even restrict $B_{j}$ to a fixed collection of subsets of $Y_{j}$ of finite $\lambda_{j}$-measure, provided the latter generates the $\sigma$-field associated with $Y_{j}$ and, moreover, is invariant under finite intersections. If $Y_{j}=\mathbf{R}^{m}$ and $\lambda_{j}$ is locally finite it would be sufficient to let $B_{j}$ be any compact interval parallel to the coordinate axes.

6.22. Richness. We will say that the measure space $(X, \lambda)$ is strongly rich relative to the given set of mappings $\pi=\left\{\pi_{j} ; j \in J\right\}$ if the following is true. Namely, we want, for each subset $D$ of $X$ with $0<\lambda(D)<\infty$, that there exists a nonzero bounded function $\theta: X \rightarrow \mathbf{R}$ supported by $D$, such that the signed measure $d \eta=\theta d \lambda$ has all its marginals $\pi_{j} \eta \quad(j \in J)$ equal to zero.

For any $1 \leq k \leq \infty$, we will say that $(X, \lambda)$ is $k$-rich (relative to $\pi$ ) if and only if, for any $\phi \in M_{0}$, not of the special form $\phi=1_{S}$ with $S$ as a set of $\pi$-uniqueness, there exist at least $k$ distinct subsets $T$ of $X$ such that $1_{T} \in M_{00}(\phi)$, equivalently, such that $1_{T} d \lambda$ has the same $\pi$-marginals as $\phi d \lambda$. As an equivalent definition, (see the first remark in 5.2), in order that $(X, \lambda)$ be $k$-rich (relative to $\pi$ ) it is necessary and sufficient that the set $M_{00}(\phi)$ contains at least $k$ distinct indicator functions, for each choice of the nonindicator function $\phi \in M_{0}$. 
Assuming Condition $\mathrm{L}$ and using (6.19), one easily verifies that $(X, \lambda)$ is strongly rich relative to $\pi$ if and only if $\left(X, \lambda, G_{\pi}\right)$ is strongly rich (in the sense of Definition 5.5), if and only if $\left(X, \lambda, F_{\pi}\right)$ is strongly rich. Similarly, if Condition L holds then $M_{00}(\phi)=M_{0}(\phi)$ for all $\phi \in M_{0}$, hence, for any $1 \leq k \leq \infty,(X, \lambda)$ is $k$-rich relative to $\pi$ if and only if $\left(X, \lambda, G_{\pi}\right)$ is $k$-rich if and only if $\left(X, \lambda, F_{\pi}\right)$ is $k$-rich.

6.23. Theorem. Suppose that $(X, \lambda)$ is strongly rich relative to $\pi$. Then $(X, \lambda)$ is nonatomic and, moreover, $\infty$-rich relative to $\pi$. In particular, each $\pi$-uniqueness function $\phi \in M_{0}$ must be an indicator function $\phi=1_{S}$.

6.24. Proof. First consider the situation that Condition L is satisfied, (as is true when $\lambda$ is finite). Then the different richness properties relative to $\pi$ are equivalent to the corresponding richness property relative to $\left(X, \lambda, G_{\pi}\right)$. From Remark 5.8, since $\left(X, \lambda, G_{\pi}\right)$ is both regular and strongly rich, it follows that $(X, \lambda)$ is nonatomic. Since each $f \in G_{\pi}$ is $\lambda$-integrable, Theorem 5.15 now implies that $\left(X, \lambda, G_{\pi}\right)$ is $\infty$-rich.

We now consider the general case. Since $(X, \lambda)$ is $\sigma$-finite, one can write $X$ as the union of at most countably many disjoint sets $X_{r}$ satisfying $0<\lambda\left(X_{r}\right)<$ $\infty$. Let $\lambda_{r}$ and $\pi^{r}$ be the restriction of $\lambda$ and $\pi=\left\{\pi_{j} ; j \in J\right\}$ to $X_{r}$. It is obvious that $\left(X_{r}, \lambda_{r}\right)$ is strongly rich relative to $\pi^{r}$. From what we proved already, it follows that $\left(X_{r}, \lambda_{r}\right)$ is nonatomic and, moreover, $\infty$-rich relative to $\pi^{r}$.

Let $\phi \in M_{0}$ be a fixed nonindicator function. We must prove that $M_{00}(\phi)$ contains infinitely many indicator functions. Let $\phi_{r}=\phi 1_{X_{r}}$ be the restriction of $\phi: X \rightarrow[0,1]$ to $X_{r}$. For each $r$, since $\left(X_{r}, \lambda_{r}\right)$ is $\infty$-rich relative to $\pi^{r}$, we have that either $\phi_{r}$ is an indicator function already or else there exist infinitely different indicator functions $\psi_{r}$ supported by $X_{r}$, such that $\psi_{r} d \lambda$ has the same projections as $\phi_{r} d \lambda$ relative to $\pi^{r}$, and, thus, relative to $\pi$. It follows that there are infinitely many different indicator functions $\psi: X \rightarrow\{0,1\}$ such that $\psi d \lambda$ has the same projections as $d \mu_{\phi}=\phi d \lambda$ relative to $\pi$, which is the desired result. Note that we are not excluding the possibility that some or all of the marginals $\pi_{j} \mu_{\phi}$ fail to be $\sigma$-finite.

6.25. Factorizations. For each $j \in J$, let $\sigma_{j}: Y_{j} \rightarrow Z_{j}$ be a (measurable) map with domain $Y_{j}$ and put $\tau_{j}=\sigma_{j} \pi_{j}$, thus, $\tau_{j}: X \rightarrow Z_{j}$. Let $\sigma=\left\{\sigma_{j} ; j \in J\right\}$ and $\tau=\left\{\tau_{j} ; j \in J\right\}$. We will say that the system $\tau$ is derived from the system $\pi$. It is useful to visualize the systems $\pi, \sigma$, and $\tau$ also as maps $\pi: X^{*} \rightarrow Y^{*}$; $\sigma: Y^{*} \rightarrow Z^{*}$, and $\tau: X^{*} \rightarrow Z^{*}$, with $X^{*} ; Y^{*}$ or $Z^{*}$, respectively, as the disjoint union of the $X_{j}=X ; Y_{j}$ or $Z_{j}(j \in J)$, respectively. This map $\tau$ is precisely the composition $\tau=\sigma \pi$ of the maps $\sigma$ and $\pi$.

Let us denote the set $M_{00}(\phi)$, interpreted relative to the system of projections $\pi$ or $\tau$, respectively, as $M_{\pi}(\phi)$ or $M_{\tau}(\phi)$, respectively. An important property is that $M_{\tau}(\phi) \supset M_{\pi}(\phi)$, for all $\phi \in M_{0}$. In fact, let $\psi \in M_{\pi}(\phi)$, that is, $\psi d \lambda$ has the same $\pi$-projections as $\phi d \lambda$. But then they also have the same 
$\tau$-projections, hence, $\psi \in M_{\tau}(\phi)$. In particular, $M_{\tau}(\phi)=\{\phi\}$ implies $M_{\pi}(\phi)=$ $\{\phi\}$.

In other words, if $\phi \in M_{0}$ is a $\tau$-uniqueness function then $\phi$ is also a $\pi$ uniqueness function. As a simpler proof, let $\phi \in M_{0}$ and $d \mu=\phi d \lambda$ and suppose one knows all the images $\nu_{j}=\pi_{j} \mu(j \in J)$. Then one also knows all the images $\tau_{j} \mu=\sigma_{j} \nu_{j}(j \in J)$ and thus $\mu$ itself, as soon as $\phi$ is a $\tau$ uniqueness function. The following is a result in the opposite direction.

6.26. Theorem. Let $\tau=\sigma \pi$ be any system of projections which can be derived from $\pi$ as above.

(i) If $(X, \lambda)$ is strongly rich relative to $\pi$ then it is also strongly rich relative to $\tau$.

(ii) For any fixed $1 \leq k \leq \infty$, if $(X, \lambda)$ is $k$-rich relative to $\pi$, (as defined in 6.22), then $(X, \lambda)$ is also $k$-rich relative to $\tau$.

6.27. Proof. As to (i), simply note that if a measure $d \eta=\theta d \lambda$ on $X$ is such that $\pi_{j} \eta$ vanishes, for all $j \in J$, then also $\tau_{j} \eta=\sigma_{j}\left(\pi_{j} \eta\right)$ vanishes, for all $j \in J$. As to (ii), let $\phi \in M_{0}$ be a nonindicator function and let $M_{\pi}(\phi)$ and $M_{\tau}(\phi)$ be as above. It is given that $M_{\pi}(\phi)$ contains at least $k$ distinct indicator functions, and we want to establish this same property for $M_{\tau}(\phi)$. But this is obvious since $M_{\tau}(\phi) \supset M_{\pi}(\phi)$.

6.28. Application. Here, we take $X=\mathbf{R}^{n}$ with $n \geq 2$, supplied with a nonzero $\sigma$-finite reference measure $\lambda$ which is absolutely continuous relative to $n$ dimensional Lebesgue measure $m_{n}(d x)=d x$. Equivalently, $\lambda(d x)=q(x) d x$, with $q: \mathbf{R}^{n} \rightarrow \mathbf{R}$ as any fixed nonzero function, such that $0 \leq q(x)<\infty$. If $q(x)$ is supported by a subset $B$ of $\mathbf{R}^{n},\left(q(x)=0\right.$ if $x \notin B$; e.g., $\left.q=1_{B}\right)$ then, for all practical purposes, one is in the case $X=B$.

We further choose $\pi=\left\{\pi_{j} ; j \in J\right\}$ as a finite collection $\pi=\left\{\pi_{1}, \ldots, \pi_{N}\right\}$ of central projections in $\mathbf{R}^{n}$. Here, the central projection $\pi_{j}$ is essentially determined by its center $P_{j}$. As to the function $\pi_{j}: \mathbf{R}^{n} \rightarrow Y_{j}$, one may choose $Y_{j}$ as any fixed hyperplane $\mathbf{R}^{n}$ not containing the point $P_{j}$. The precise choice of $Y_{j}$ is irrelevant. In fact, whatever the choice of $Y_{j}$, we ha:-e for each $\phi \in M_{0}$ that the $\pi_{j}$-projection $\nu_{j}=\pi_{j} \mu$ of the measure $d \mu=\phi d \lambda$ on $\mathbf{R}^{n}$ is fully determined by the associated function

$$
h_{j}(z)=\int_{-\infty}^{\infty} \phi\left(P_{j}+t z\right) q\left(P_{j}+t z\right) d t, \text { thus, } h_{j}(\rho z)=h_{j}(z) /|\rho|
$$

$\left(x \in \mathbf{R}^{n}, \rho \in \mathbf{R}\right)$. We will also allow one or more centers $P_{j}$ to be points at infinity in which case $\pi_{j}$ is to be interpreted as a parallel projection along lines of direction $P_{j}$. One may choose $Y_{j}$ as any fixed hyperplane in $\mathbf{R}^{n}$ not parallel to $P_{j}$. For such a parallel projection, $(6.29)$ is to be replaced by

$$
H_{j}(z)=\int_{-\infty}^{\infty} \phi\left(z+t w_{j}\right) q\left(z+t w_{j}\right) d t \quad\left(z \in \mathbf{R}^{n}\right),
$$


with $w_{j}$ as a fixed nonzero vector in $\mathbf{R}^{n}$ having direction $P_{j}$. Note that $H_{j}(z)$ is constant along lines of direction $P_{j}$. The following result was established in $[5,12]$.

6.31. Theorem. Choose $X=\mathbf{R}^{n}$ and $\lambda(d x)=q(x) d x$. Then $(X, \lambda)$ is strongly rich relative to any finite system $\pi=\left\{\pi_{1}, \ldots, \pi_{N}\right\}$ of central projections in $\mathbf{R}^{n}$.

6.32. Theorem. Let $X=\mathbf{R}^{n}$ and $\lambda(d x)=q(x) d x$. One has from Theorems 6.26 and 6.31 that $(X, \lambda)$ is strongly rich relative to any system $\tau=$ $\left\{\tau_{1}, \ldots, \tau_{N}\right\}$ of projections which can be derived (as in 6.25) from a finite system $\pi=\left\{\pi_{1}, \ldots, \pi_{N}\right\}$ of central projections in $\mathbf{R}^{n}$. This includes each system $\tau$ consisting of finitely linear maps $\tau_{j}: \mathbf{R}^{n} \rightarrow Z_{j}$, where $Z_{j}$ is a linear space such that $\operatorname{dim} Z_{j} \leq n-1$.

It follows from Theorem 6.23 that $(X, \lambda)$ is also $\infty$-rich relative to any such $\pi$ or $\tau$. In particular, each $\pi$-uniqueness function is an indicator function. Moreover, if $0 \leq \phi \leq 1$ is not a $\pi$-uniqueness function then there exist infinitely many distinct indicator functions $1_{S}$ such that $1_{S} \in M_{00}(\phi)$; that is, $1_{S} d \lambda$ has the same $\pi$-projections as $\phi d \lambda$. Similarly when $\pi$ is replaced by any derived system $\tau$.

6.33. The classical case. By the way, the above results do not carry over to a countable system $\pi$ of central projections. For instance, consider a nonindicator function $0 \leq \phi \leq 1$ on $\mathbf{R}^{2}$ of compact support and let $\lambda=m_{2}$. Since the Fourier transform of $\phi$ is an entire function, one easily sees that the measure $d \mu_{\phi}=\phi d \lambda$ is uniquely determined by $\left\{\pi_{j} \mu_{\phi} ; j=1,2, \ldots\right\}$ with $\pi=\left\{\pi_{j}\right.$; $j=1,2, \ldots\}$ as any fixed system consisting of countably many distinct parallel projections in $\mathbf{R}^{2}$. In particular, the corresponding set $M_{00}(\phi)$ does not contain any indicator function. That is, there exists no measure of the form $d \mu_{S}=1_{S} d \lambda$ such that $\pi_{j} \mu_{S}=\pi_{j} \mu_{\phi}$, for all $j$.

Consider now the so-called classical case where $X=\mathbf{R}^{n} \quad(n \geq 2)$ and $d \lambda=$ $q d x$ are as above while $\tau=\left\{\tau_{1}, \ldots, \tau_{n}\right\}$ with $\tau_{j}: \mathbf{R}^{n} \rightarrow \mathbf{R}$ as the ('onedimensional') linear map $\tau_{j} x=x_{j}$, which maps $x=\left(x_{1}, \ldots, x_{n}\right) \in \mathbf{R}^{n}$ to its $j$ th coordinate $(j=1, \ldots, n)$. This system $\tau$ has all the indicted properties. Presently, we have for each $\phi \in M_{0}$ that

$$
F_{\pi}(\phi)^{*}=\left\{f(x)=g_{1}\left(x_{1}\right)+\cdots+g_{n}\left(x_{n}\right): \int_{R^{n}}\left|g_{j}\left(x_{j}\right)\right| \phi(x) q(x) d x<\infty\right\}
$$

$(j=1, \ldots, n)$. An open and difficult problem is to describe the structure of all sets $S$ of uniqueness. The only known way of constructing a set of uniqueness is in the form of an additive set or generalized additive set, (or a certain even more general sets where the construction in 3.10 is extended to any denumerable ordinal number, see [11] for details).

6.35. The following comments all refer to the classical case above. Much more can be said when $n=2$ and $q \equiv 1$ thus $\lambda=m_{2}$. Namely, it turns out that 
then a subset $S$ of $\mathbf{R}^{2}$ satisfying $\lambda(S)<\infty$ is a set of uniqueness if and only if it is additive, that is, of the form $g_{1}\left(x_{1}\right)-g_{2}\left(x_{2}\right) \geq 0$, (up to a set of $m_{2}$-measure zero). This important result is due to Fishburn et al. [3], and Kuba and Volcic $[16,18]$, each making an essential use of results due to Lorentz [17].

We conclude that, in the latter $n=2$ case, each special generalized additive set $S$ as in (6.11) satisfying $\lambda(S)<\infty$ must already be additive. This seems to be related to the fact that the functions $f_{q} \in F_{\pi}(S)^{*}$ in (6.11) are far from unique when $n=2$. After all, $f(x)=g_{1}\left(x_{1}\right)-g_{2}\left(x_{2}\right) \geq 0$ is equivalent to $\theta\left(g\left(x_{1}\right)\right)-\theta\left(g_{2}\left(x_{2}\right)\right) \geq 0$, for each choice of the strictly increasing function $\theta: \mathbf{R} \rightarrow \mathbf{R}$. More general results for the classical case $n=2$ will be presented in a subsequent paper, where we allow for an arbitrary function $q(x)$ and the possibility that $\lambda(S)=\infty$.

The analogous result is false for the classical case with $n \geq 3$. More precisely, if $n \geq 3$ then there exists [11] a special 1-additive set $S$, (that is, a set of the form (6.11) with $s=1$ and $\lambda(Z)=0$ ), which is not additive, (see 3.23 and 3.24 for related remarks).

In more detail, let $n=3$ for simplicity and consider $K=[0,2) \times[0,2) \times$ $[0,1)$, a bounded subset of $\mathbf{R}^{3}$. Choose $q=1_{K}$, that is, $\lambda$ is a Lebesgue measure $m_{3}$ restricted to $K$, thus, one is essentially in the case $X=K$. Further choose $S$ as the set of all $x \in K$ such that either $f_{0}(x) \geq 0$ or $f_{0}(x)=0$ and $f_{1}(x) \geq 0$, where

$$
f_{0}(x)=\theta\left(x_{1}\right)+\theta\left(x_{2}\right) \text { and } f_{1}(x)=x_{1}+x_{2}+x_{3}-2 .
$$

Here $\theta(u)=0$ for $0 \leq u<1$ and $\theta(u)=3 / 2-u$ for $1 \leq u<2$. In particular, $\lambda(Z)=0$ with $Z$ as in (6.12) and $s=1$. The resulting special 1 -additive set $S$ is the union of a prism, a tetrahedron and two rectangular blocks. Much more general pairs $f_{0}, f_{1}$ work equally well. The only problem here is to prove that the latter set $S$ is not additive. This is done [11] by showing that there does not exist any triplet of measurable functions $h_{1}, h_{2}, h_{3}$, such that, for almost all $x \in K$, one has $\sum_{j} h_{j}\left(x_{j}\right) \geq 0$ if and only if $x \in S$.

6.36. Optimization. Let $\left(X, \lambda, G_{\pi}\right)$ be as above, thus $G_{\pi}$ is determined as in (6.20) by a given system $\pi$ of projections $\pi_{j}: X \rightarrow Y_{j}^{\pi}(j \in J)$ which is assumed to satisfy Condition $\mathrm{L}$, see 6.16 . In particular, $\left(X, \lambda, G_{\pi}\right)$ is regular and, moreover, each $f \in G_{\pi}$ is $\lambda$-integrable.

Let further $h: X \rightarrow R$ be a given $\lambda$-integrable function. Quite a common problem (see [6, Appendix; 8, 9, and 10]) is the study of $\sup _{\mu} \int h d \mu$, when $\mu$ can be any measure on $X$ satisfying $\mu \leq \lambda$ and having preassigned marginals $\nu_{j}=\pi_{j} \mu \quad(j \in J)$. If the supremum is achieved by $d \mu=\phi d \lambda$ (with $\phi \in M_{0}$ ) we will say that $\phi$ is optimal.

Here, we are exactly in the setting of 5.23 but with $F$ replaced by $G_{\pi}$. It follows that optimal functions $\phi$ always exist. One also would like to know whether some optimal $\phi$ is an indicator function and to what extent $\phi$ is unique. 
Suppose that $\left(X, \lambda, G_{\pi}\right)$ is strongly rich (and thus nonatomic). This assumption is satisfied, for instance, for the example in 6.28 (see Theorem 6.31) and, hence, for the classical case as described in 6.33. Then it follows from the remarks in 5.23 that either there is only one optimal function, necessarily an indicator function, or else there exist infinitely many indicator functions each of which is an optimal function.

\section{REFERENCES}

1. N. I. Ahiezer and M. Krein. Some questions in the theory of moments, Vol. 2. Transl. Math. Monos., Amer. Math. Soc, Providence, R.I.. 1962.

2. W. A. Carrington, Moment problems and ill-posed operator equations with convex constraints, Ph.D. Thesis, Washington Univ., St. Louis, 1982.

3. P. C. Fishburn, J. C. Lagarias, J. A. Reeds and L. A. Shepp, Sets uniquely determined by projections on axes I. Continuous case, SIAM J. Appl. Math. (to appear).

4. __ Sets uniquely determined by projections on axes II. Discrete case, Preprint, $13 \mathrm{pp}$.

5. S. Gutmann, J. H. B. Kemperman, J. A. Reeds and L. A. Shepp, Existence of probability measures with given marginals. Ann. Probab. (to appear).

6. K. Jacobs, Measure and integral. Academic Press. New York, 1978.

7. S. Karlin and W. J. Studden, Tchebycheff sy'stems: with applications in analysis and statistics, Interscience, New York. 1966.

8. J. H. B. Kemperman, On a class of moment problems, Proc. Sixth Berkeley Sympos. Math. Stat. and Prob., vol. 2, Univ. of California Press. Berkeley, 1972, pp. 101-126.

9. $\ldots$, On the role of duality in the theory of moments, Semi-infinite Programming and Applications (A. V. Fiacco and K. O. Kortenek, eds.), Lecture Notes in Economics and Mathematical Systems, vol. 215. Springer-Verlag. New York, 1983, pp. 63-92.

10. __ Geometry of the moment problem. Moments in Mathematics (H. J. Landau, ed.), Proc. Sympos. Appl. Math., Vol. 37. Amer. Math. Soc.. Providence, R.I., 1987, pp. 16-53.

11. __ Sets of uniqueness and s!stems of inequalities having a unique solution. Pacific $\mathbf{J}$. Math. (to appear).

12. __ On the lack of uniqueness when reconstructing a set from finitely many central projections (in preparation).

13. J. F. C. Kingman and A. P. Robertson. On a theorem of Lyapunor, J. London Math. Soc. 43 (1968), 347-351.

14. M. G. Krein, The ideas of P. L. Cebyer and A. A. Markor in the theory of limiting values of integrals and their further development. Uspekhi Mat. Nauk 6 (1951). 3-130: English transl., Amer. Math. Soc. Transl. (2) 12 (1959), 1-121.

15. M. G. Krein and A. A. Nudel' man, The Markov moment problem and extremal problems, Transl. Math. Monos., vol. 50. Amer. Math. Soc., Providence, R.I., 1977.

16. A. Kuba and A. Volcic, Characterization of measurable plane sets, which are reconstructible from their projections, Inverse Problems 4 (1988), 513-527.

17. G. G. Lorentz, A problem of plane measure. Amer. J. Math. 71 (1949), 417-426.

18. A. Volcic, On a class of plane sets, which can be reconstructed from two Steiner symmetrizations, Manuscript.

Department of Statistics, Rutgers University, New Brunswick. New Jersey 08903 